\title{
Multi-scale analysis of the interaction in ultra-long carbon nanotubes and bundles
}

\author{
Mengxiong Liu ${ }^{\mathrm{a}, \mathrm{b}, 1}$, Xuan Ye $\mathrm{e}^{\mathrm{a}, \mathrm{c}, 1}$, Yunxiang Bai ${ }^{\mathrm{d}, 1}$, Rufan Zhang ${ }^{\mathrm{d}}$, Fei Wei ${ }^{\mathrm{d}}$, \\ Xide $\mathrm{Li}^{\mathrm{a}} \mathrm{b}, *$ \\ a Department of Engineering Mechanics, Applied Mechanics Lab, Tsinghua University, Beijing 100084, China \\ ${ }^{\mathrm{b}}$ Center for Nano and Micro Mechanics, Tsinghua University, Beijing 100084, China \\ ' State Key Lab. of Nonlinear Mechanics, Institute of Mechanics, Chinese Academy of Sciences, Beijing 100190, China \\ ${ }^{\mathrm{d}}$ Beijing Key Laboratory of Green Chemical Reaction Engineering and Technology, Department of Chemical Engineering, Tsinghua \\ University, Beijing 100084, China
}

\section{A R T I C L E I N F O}

\section{Article history:}

Received 13 January 2020

Revised 12 May 2020

Accepted 22 May 2020

Available online 30 May 2020

\section{Keywords:}

Ultra-long CNT/CNTB

Micro/nanoscale mechanical testing system (m/n-MTS)

Multi-scale analysis

Boundary effects

Dynamic friction

\begin{abstract}
A B S T R A C T
Ultralow inter-wall interaction is realized in centimeter-long double-walled carbon nanotube (DWCNT) (Zhang, R. et al. Nat. Nanotech. 8, 912-916 (2013)). To further illustrate the mechanism of this special phenomenon, theoretical analysis combining with the experimental test is conducted, where study of the inter-tube interaction in the carbon nanotube bundles (CNTBs) is also included in this paper. In experiment, nanomanipulation difficulties of the ultra-long carbon nanotube (CNT)/CNTB is overcome by establishing a micro/nanoscale mechanical testing system (m/n-MTS), based on which the inter-wall/intertube interaction is firstly in-situ measured under optical microscope (OM). In theory, according to the bottom-up approach, a multi-scale model is developed specially, by which results obtained can be directly compared with the experimental data. The pull-out force consists of three parts: the boundary effect, the configuration force and the dynamic friction. The boundary effect can account for more than $70 \%$ when the pull-out velocity equals to $1 \mu \mathrm{m} / \mathrm{s}$. The configuration force is greatly enhanced for the CNTB because of the commensurate configuration in the circumferential direction. The dynamic friction shows linear dependence on the pull-out velocity and relates to the overlapped area, which can be manifested when the pull-out velocity reaches to a dozen $\mu \mathrm{m} / \mathrm{s}$ for the ultra-long DWCNT. The dynamic friction coefficient is proved to be 0.29 multiple of critical value based on the experiment data. The pull-out force in the CNTB is found far less than that in the DWCNT due to the greatly reduced contact area. Moreover, it can be deduced that the dynamic friction will show the same order of magnitude as the contribution from the boundary when the pull-out velocity equals to $10 \mu \mathrm{m} / \mathrm{s}$ or the length is $10 \mathrm{~mm}$. This research reveals the underlying mechanism of the interaction, especially for the dynamic friction force exhibited in the ultra-long CNT/CNTB, and can provide help for designing devices with ultralow friction on macroscale.
\end{abstract}

(c) 2020 Elsevier Ltd. All rights reserved.

\footnotetext{
* Corresponding author.

E-mail address: lixide@tsinghua.edu.cn (X. Li).

1 These authors contribute equally to this work.
} 


\section{Introduction}

On the micro/nano scale, friction and wear can be greatly enhanced because of the extremely high surface-to-volume ratio, which are considered as the bottlenecks to restrict the development of Micro/Nano Electro-Mechanical Systems (M/NEMS) (Urbakh and Meyer, 2010). Double-walled carbon nanotubes, especially for the centimeter-long ones, are proved to be with ultralow inter-wall interaction (Rivera et al., 2003; Kis et al., 2006; Zhang et al., 2013a), which really attracts researchers' attention because friction and wear can be maximally reduced ranging from nano- to macroscale. Furthermore, DWCNTs also have excellent mechanical and electronic properties (Yu et al., 2000a; Guo and Guo, 2003). Thus, they can be considered as the candidates for fabricating M/NEMS such as nano-motors, nano-oscillator and ultrasensitive sensors (Riedo et al., 2001; Fennimore et al., 2003; Zheng and Jiang, 2002). Moreover, the tensile strength of carbon nanotube bundles (CNTBs) is 1-2 orders of magnitude lower than that of a single carbon nanotube (CNT) because the typical length of CNT segments is much less than that of the entire bundle and the tubes are connected by the weak van der Waals (vdW) interaction (Lu et al., 2012; Liu et al., 2011; Yakobson et al., 2000). However, the tensile strength of CNTBs assembled by several ultra-long CNTs can reach $80 \mathrm{GPa}$ by releasing the non-uniform initial strains through the synchronous tightening and relaxing strategy (Bai et al., 2018). The weak inter-tube interaction plays an important role in this method, which is similar to the lubricants in ropes, helping to adjust CNTBs with uniformly aligned components. Therefore, having an in-depth study of the inter-wall interaction of ultra-long DWCNTs and the inter-tube interaction of ultra-long CNTBs is of great significance for promoting their applications in M/NEMS and fabricating ultra-strong fibers.

Due to the difficulties in performing nanomanipulation, experimental characterization of CNT at nanoscale is hardly realized in the current stage and only a few experimental studies are reported on the inter-wall sliding behaviors of DWCNT (Kis et al., 2006; Yu et al., 2000b; Cumings and Zettl, 2000). However, due to the presence of defects or deformations, the measured inter-wall interaction is generally much higher than that of the theoretical predictions (Yu et al., 2000a; Cumings and Zettl, 2000; Kis et al., 2006;). Moreover, although these experimental tests are operated in scanning electron microscope (SEM), the fracture behaviors cannot be directly observed and the occurrence of the sliding events can only be inferred by the displacement evolution of cantilever tip with time, for example, in Yu's experiment (Yu et al., 2000b). Recently, benefitting from the breakthrough of preparing defect-free CNT with several millimeters in length (Zhang et al., 2013a; Zhang et al., 2017a) and the optical visualization realized by decorating $\mathrm{TiO}_{2}$ on the surface of the CNT, the damage from electron microscopy can be avoided and the ultralow inter-wall interaction is measured in-situ. However, a series of discontinuous loading values are obtained because the force sensor is designed by characterizing the deformation of a silicon nanorod. In general, although the nanomanipulation seems easier to be realized for characterizing the inter-wall/inter-tube interaction in ultra-long DWCNTs/CNTBs due to the millimeter scale in length, new experiment challenges appear because they are multi-scale structures with macroscale in length and nanoscale in circumferential direction.

Owing to the development of computation tools, including density functional theory (DFT), molecular dynamics (MD) and molecular statics (MS), various theoretical studies have been carried on the inter-wall interaction of DWCNT (Guo et al., 2003; Liu and Zhang, 2011; Servantie and Gaspard, 2006b). In the MS simulation or MD with low temperature, the pull-out force is computed as the numerical derivative of the increased potential energy with respect to displacement (Akita and Nakayama, 2003a; Akita and Nakayama, 2005; Guo et al., 2005). As a result, when pulling out the inner tube from the outer one, the force originated from vdW interaction is only related to the relative position of inner and outer tubes while is independent to time. Especially, it is independent with the overlapped area for the incommensurate configuration (Guo et al., 2003; Li et al., 2010; Guo and Gao, 2005; Yuan and Wang, 2017). In the MD simulation when the temperature is considered, the total energy of the system is no longer conservative and energy dissipation caused by phonons should be considered (Guo et al., 2003; Zhang et al., 2004; Zhao et al., 2003; Omata et al., 2005; Rivera et al., 2005). The longer interface allows absorb more energy from the traveling phonon (Prasad and Bhattacharya, 2017), but in fact, the dynamic friction force is usually ignored in the theoretical models because it is substantially smaller than the force caused by vdW interaction (Zheng et al., 2002; Zheng and Jiang, 2002). However, these traditional tools are found cannot be applied to compute the inter-wall interaction of ultra-long DWCNT due to the limitation of computing time and space. Moreover, some new questions may arise. For example, whether the conclusions obtained at nanoscale are suitable for the ultra-long DWCNTs, whether the dynamic force can still be ignored for ultra-long DWCNTs and whether new phenomenon will appear in the inter-wall interaction for DWCNTs on macroscale. Therefore, it is an urgent need to develop new computation method to explain the results and the new phenomenon obtained in the experiment of ultra-long DWCNTs.

According to the previous study, the inter-tube interaction plays an important role in the tensile strength of CNTBs (Yakobson et al., 2000; Lu et al., 2012; Yu et al., 2000a). Various efforts are devoted to study the inter-tube interaction in CNTBs (Filleter et al., 2012; Mirzaeifar et al., 2015; Paci et al., 2016), where the boundary effects, defects, twisting and chemically functionalized modification are included. According to Filleter's report (Filleter et al., 2012), the normalized pull-out force per CNT-CNT interaction $(1.7 \pm 1.0 \mathrm{nN})$ is found to be considerably higher than the molecular mechanics (MM)-based predictions for bare CNTs $(0.3 \mathrm{nN})$, which mainly attributes to the dissipation, surface energy and topological defects. However, the inter-tube interaction in the bare and pristine CNTBs is rarely reported due to the difficulties in preparation process of continuous, horizontally aligned and defect-free materials. Recently, due to the preparation breakthrough of the ultra-long CNTs, the ultra-long CNTBs are synthesized (Bai et al., 2018; Zhang et al., 2017b). Therefore, this supplies the opportunities to investigate the inter-tube interaction of the bare and pristine CNTB in experiment and some issues should be discussed. For example, whether the ultralow inter-tube interaction also exists in the ultra-long CNTBs, what differences will appear 

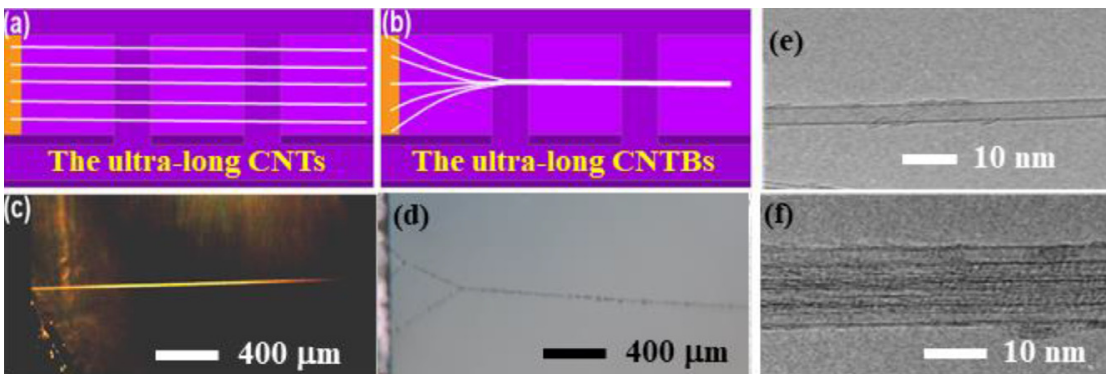

Fig. 1. . Fabrication and structural characterization of ultra-long CNTs/CNTBs. (a) and (b) show the preparation method of ultra-long CNTs/CNTBs. (c) and (d) illustrate the ultra-long CNTs/CNTBs decorated with $\mathrm{TiO}_{2}$ particles, resulting in the optical visualization. (e) and (f) are the TEM images of the ultra-long CNTs/CNTBs.

compared to the inter-wall interaction in ultra-long DWCNTs and whether new phenomenon will appear when pulling out the CNT from the ultra-long CNTBs.

Based on the considerations mentioned above, this paper carries out the study of interaction in ultra-long DWCNTs/CNTBs combining the experimental test and theoretical analysis. This paper is organized as follows. To capture the continuous loading-displacement values, an innovative micro/nanoscale mechanical testing system (m/n-MTS) is built, where a force sensor connected with the probe is used to provide the load data real time. Thus, the inter-wall/inter-tube interaction is firstly in-situ tested in optical microscope (OM) and the detailed introduction of the experimental tests is illustrated in Section. 2. Considering the limitations of the traditional computation methods to the ultra-long CNTs/CNTBs, a multi-scale model based on the bottom-up approach is developed to describe the interaction behaviors in ultra-long DWCNTs /CNTBs and to explain the mechanism behind the experimental results, which is described in details in Section. 3. In Section. 4, the developed multi-scale model is verified by the MD simulation firstly. Then, by setting the parameters according to the experiment, the results obtained from this multi-scale model are compared with the experimental tests and the mechanisms are explained. Based on this fact, the multi-scale model is applied to make predictions of the behaviors of pulling out a single CNT from the ultra-long CNTBs. Finally, some important conclusions are obtained at the end.

\section{Experiments of interaction in the ultra-long CNTs /CNTBs}

The ultra-long CNTs are prepared through a gas-flow directed chemical vapour deposition (CVD) method and then the ultra-long CNTB is synthesized by focusing gas flow, which can assemble individual CNTs into a bundle (Zhang et al., 2013b; Liu et al., 2009) shown in Fig. 1 (a) and (b). Due to the particularity of these structures, which are several millimeters along axial direction and a few nanometers in circumferential direction, the nano sized $\mathrm{TiO}_{2}$ particles are decorated on the surface to realize the optical visualization expressed in Fig. 1 (c) and (d) (Zhang et al., 2013c). The effect of the TiO 2 particles on the structure of the CNTs/CNTBs is discussed in details in the supporting information (Note S1). Transmission Electron Microscope (TEM) images are shown in Fig. 1 (e) and (f), where the individual ultra-long CNTs are nearly parallel alignment in the ultra-long CNTBs.

Considering the structural particularity of ultra-long CNTs/CNTBs with slenderness ratio over $10^{7}$, a micro/nanoscale mechanical testing system (m/n-MTS) shown in Fig. 2 is built to investigate the inter-wall/inter-tube interaction, as has been used previously (Bai et al., 2018; Ye et al., 2017a; Ye et al., 2017b). As shown in Fig. 2 (a), the testing system consists of three parts: the computer used to the m/n-MTS and record the load and the displacement, the optical microscope (OM) used to monitor the pull-out process and the micro tensile system designed to loading. The micro tensile system is enlarged in Fig. 2 (b), where the specimen is bonded on the left side of the sample end by conductive adhesive and the micro force sensor is fixed on the right side. By adjusting the translation stage, the probe of the force sensor will be in contact with the CNTs/CNTBs by the vdW interaction. As the coordinate system defined in Fig. 2(b), the x-axis, y-axis, z-axis translation stages can move continuously along the $\mathrm{x}+/ \mathrm{x}-, \mathrm{y}+/ \mathrm{y}-, \mathrm{z}+/ \mathrm{z}$ - directions, separately. Also, the capacitive micro-force sensor is sensitive to $\mathrm{x}$-axial tension and compression forces. Therefore, as shown in Fig. 2(e), pushing (loading along $\mathrm{x}$ - direction) or pulling (along $\mathrm{x}+$ direction) a DWCNT to measure the pullout force is equivalent. Moreover, as shown in Fig. 2(f),due to the fact that the sensor probe with a radius less than $2 \mu \mathrm{m}$ makes contact with the DWCNT/CNTB by vdW forces, slip failure rarely appears at the clamping ends during tests regardless of the pushing (loading along $\mathrm{x}$ - direction) or pulling (along $\mathrm{x}+$ direction). The testing principle is shown in Fig. 2 (c), where the ultra-long CNT is similar to a stretched string and thus the load can be calculated by the formula shown in this figure. Fig. 2 (d) illustrates the testing process of pulling out the inner tube from the outer one. After the outer tube breaks, the inner tube is pulled out gradually. Then, the pulled out inner tube will retract after the external load is removed. The retraction behavior of the inner tube originates from the boundary effect and the detailed explanation is illustrated in the supporting information (Note S1 and Video 1). The displacement resolution of the translation stage is $0.5 \mathrm{~nm}$, the resolution of micro-force sensing probe is $5 \mathrm{nN}$ (FT-S100, FemtoTools, Switzerland) and the magnification range of the optical microscope is 350-3,500 $\times$ (HIScope KH-3000, Hirox, Japan). 

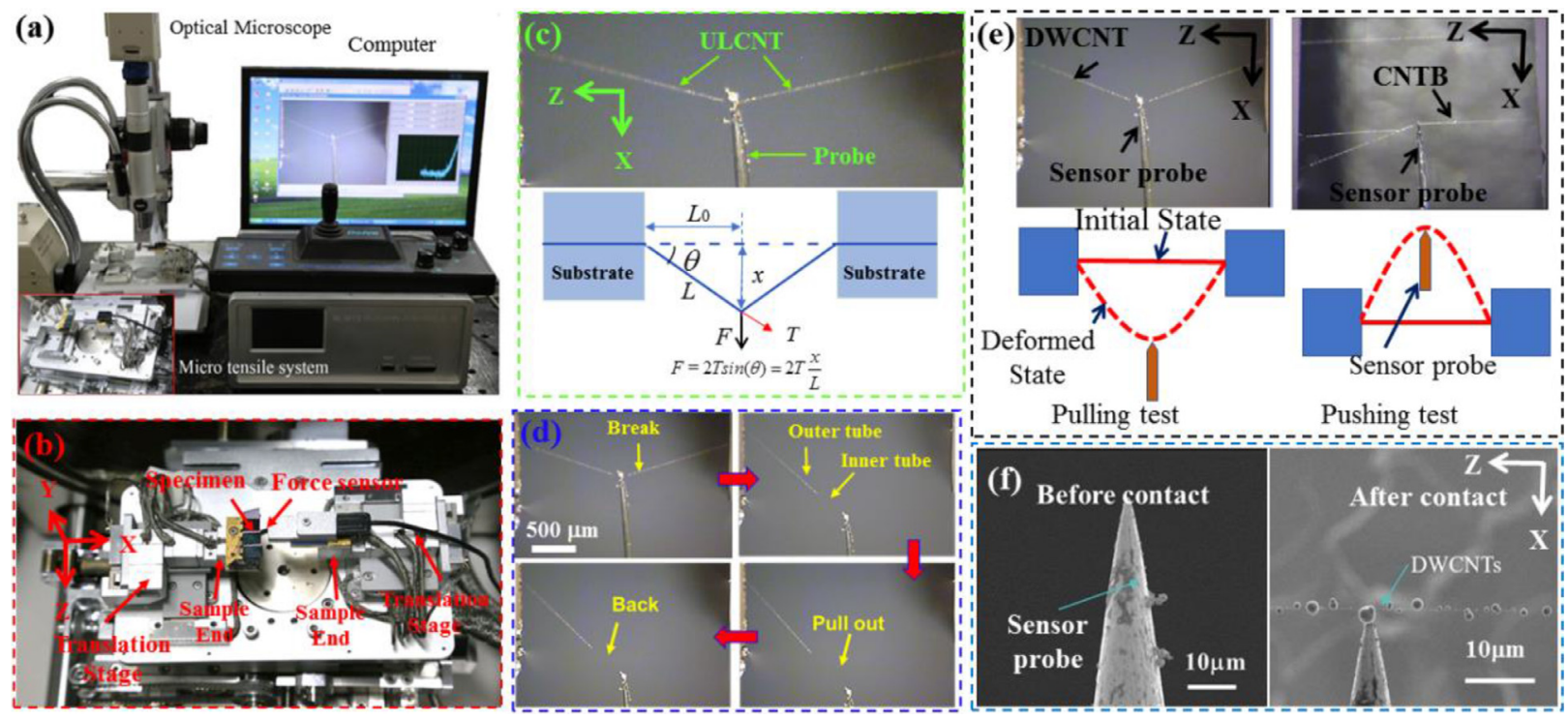

Fig. 2. The micro/nanoscale mechanical testing system (m/n-MTS) for ultra-long CNT/CNTB. (a) shows the global arrangement of the testing system, consisting of computer, optical microscope and the MTS. (b) is the enlargement and introduction of the MTS, where the coordinate system defined. (c) expresses the schematic diagram of testing principle. (d) shows the testing process of pulling out the inner tube from the outer one. (e) shows the pulling/pushing tests and the corresponding diagrams. (f) is the enlarged drawing of the sensor probe.

The initial length of the tested ultra-long DWCNT is $2 \mathrm{~mm}$, the diameter of the outer tube is $2.67 \mathrm{~nm}$ and the pullout velocity of the sensor is set as $14 \mu \mathrm{m} / \mathrm{s}$. According to the loading data $(T)$ recorded by the force senor, loading on the ultra-long DWCNT $(F)$ can be obtained by the formula in Fig. 2 (c). Fig. 3 (a) displays a grouped set of images, representing the pull-out process of the inner CNT from the outer one and the corresponding loading-displacement curve is shown in Fig. 3 (b). The pull-out process can be divided into three stages. The first stage begins with the outer tuber rapidly broken and the stretch loading drops sharply, which corresponds to the State 1 . Then, the inner tube is gradually pulled out until the right side is broken. The average force in this stage is $11.8 \mathrm{nN}$. After that, the pull-out process enters into the second stage, which corresponds to the State 2 and the right side of the inner tube cannot be found out in the optical image. After that, the left side of the inner tube is pulled out (blue line), where the distance between the probe and the visualized outer tube (white line) increases gradually. The pull-out velocity of the inner tube in this stage is $6.9-10.2 \mu \mathrm{m} / \mathrm{s}$ because the angle defined in Fig. 2 (c) varies from $29^{\circ} 51^{\prime}$ to $47^{\circ} 24^{\prime}$, resulting in $7.8 \mathrm{nN}$ of the average force. To further pull out the inner tube, the magnification of microscope is decreased, which corresponds to the third stage. The average pull-out force is $8.6 \mathrm{nN}$ when the pull-out velocity varies in $10.2-11.7 \mu \mathrm{m} / \mathrm{s}$. The fluctuation degrees in the three stages are characterized by the standard deviation of data shown in Fig. 3 (b). The pull-out force is comparable but higher than the previous values tested in the quasi-static state, including $3.1 \mathrm{nN}$ (Kis et al., 2006), $4.0 \mathrm{nN}$ (Akita and Nakayama, 2003b) and theoretical value $2 \mathrm{nN}$ (Akita and Nakayama, 2005).

The pull-out process of a CNT from the ultra-long CNTB is shown in Fig. 4. The State 1 shows the initial configuration of the CNTB consisting of three tubes marked as 1, 2 and 3 on the left side of the probe, while shown as a bundle on the right side. The initial length is $2 \mathrm{~mm}$ and diameters of the CNTs are $2.61 \mathrm{~nm}, 1.82 \mathrm{~nm}$ and 2.02 nm, respectively. The pull-out velocity of the sensor is set as $14 \mu \mathrm{m} / \mathrm{s}$. The maximum loading value is $202 \mathrm{nN}$, where the corresponding configuration is State 2. After the CNTB on left side of probe is broken and cannot be found out in the optical image, the pull-out process begins in State 3, where the stretch loading rapidly descends to near zero. Then, the core CNT (blue line) is gradually pulled out from the CNTB (white line) with the probe moving forward. As shown in Fig. 4 (b), the average pull-out force is 0.9 nN when the pull-out velocity of the core CNT is 5.6-7.2 $\mu \mathrm{m} / \mathrm{s}$ due to the variation of the angle defined in Fig. 2 (c) $\left(23^{\circ} 39^{\prime}\right.$ to $\left.31^{\circ} 05^{\prime}\right)$. The tested pull-out force value is comparable but higher than the previous reported values in the quasi-static state, including the $0.43 \mathrm{nN}$ (Benassi, et al., 2008) and $0.3 \mathrm{nN}$ (Filleter et al., 2012).

\section{The multi-scale model of the interaction in the ultra-long CNT /CNTB}

To illustrate the interaction in the ultra-long CNT/CNTB obtained from experiments, a multi-scale model is developed and shown in Fig. 5. It consists of three typical models in different scales. The atomic scale model is based on the MD method and it could reach dozens of nanometers (Fig. 5a). Then, the basic parameters of carbon nanotube and the inter-wall/intertube interaction discussed in Section 3.1 will be obtained, which can be used to construct the mesoscale model according to the coarse-grained method (Buehler and Markus, 2006). As a result, the computation scale is hundreds of nanometers (Fig. 5b), while the detailed structural information of carbon nanotube including the six-membered ring and the $\pi$ bond is 

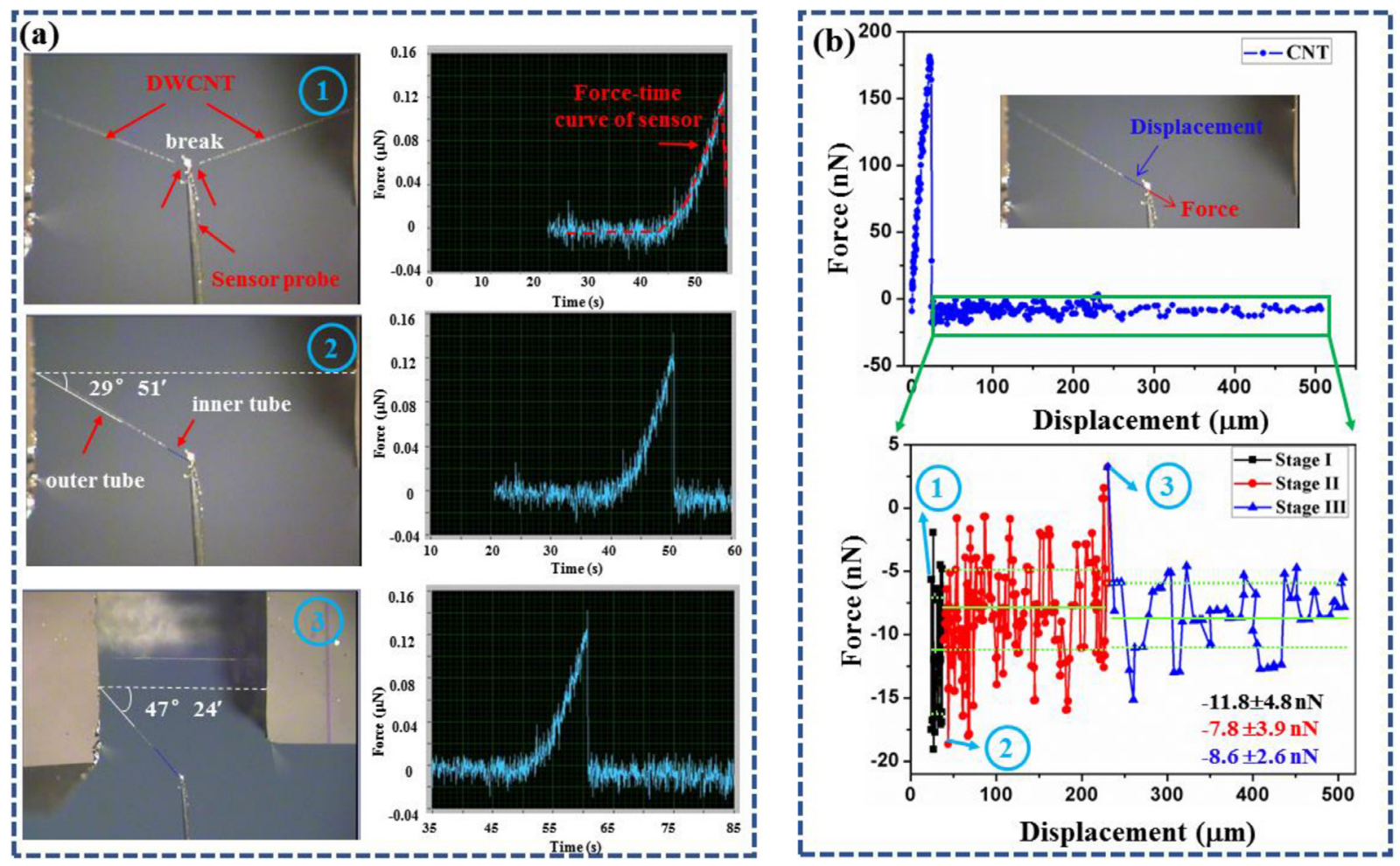

Fig. 3. The pull-out process of CNT in the ultra-long DWCNT. (a) is a set of optical images showing the pull-out process. (b) expresses the corresponding loading-displacement curve.
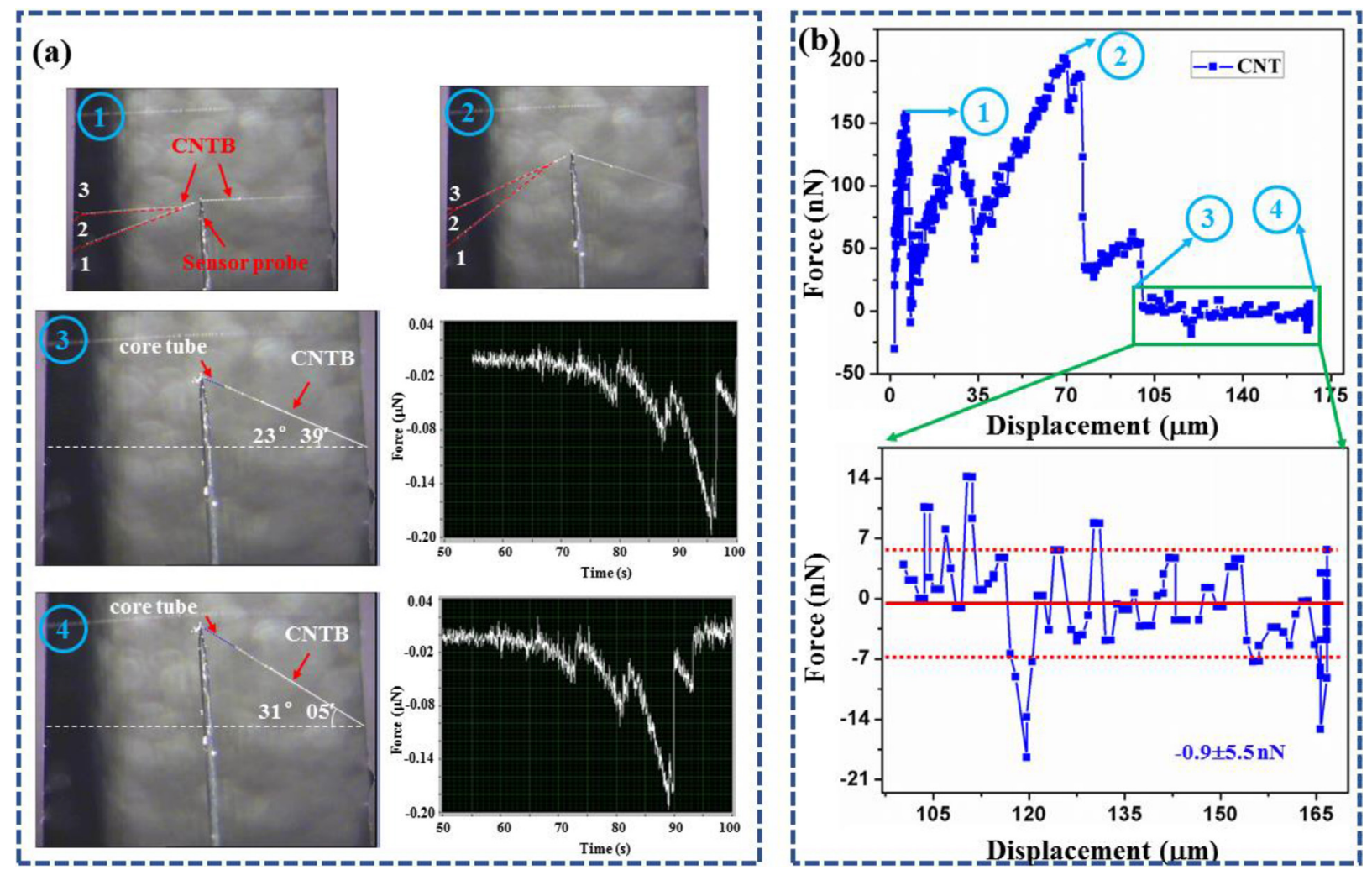

Fig. 4. The pull-out process of ultra-long CNTB in the experiment. (a) shows the pull-out process by the captured images from recorded video. (b) is the calculated loading-displacement curve. 

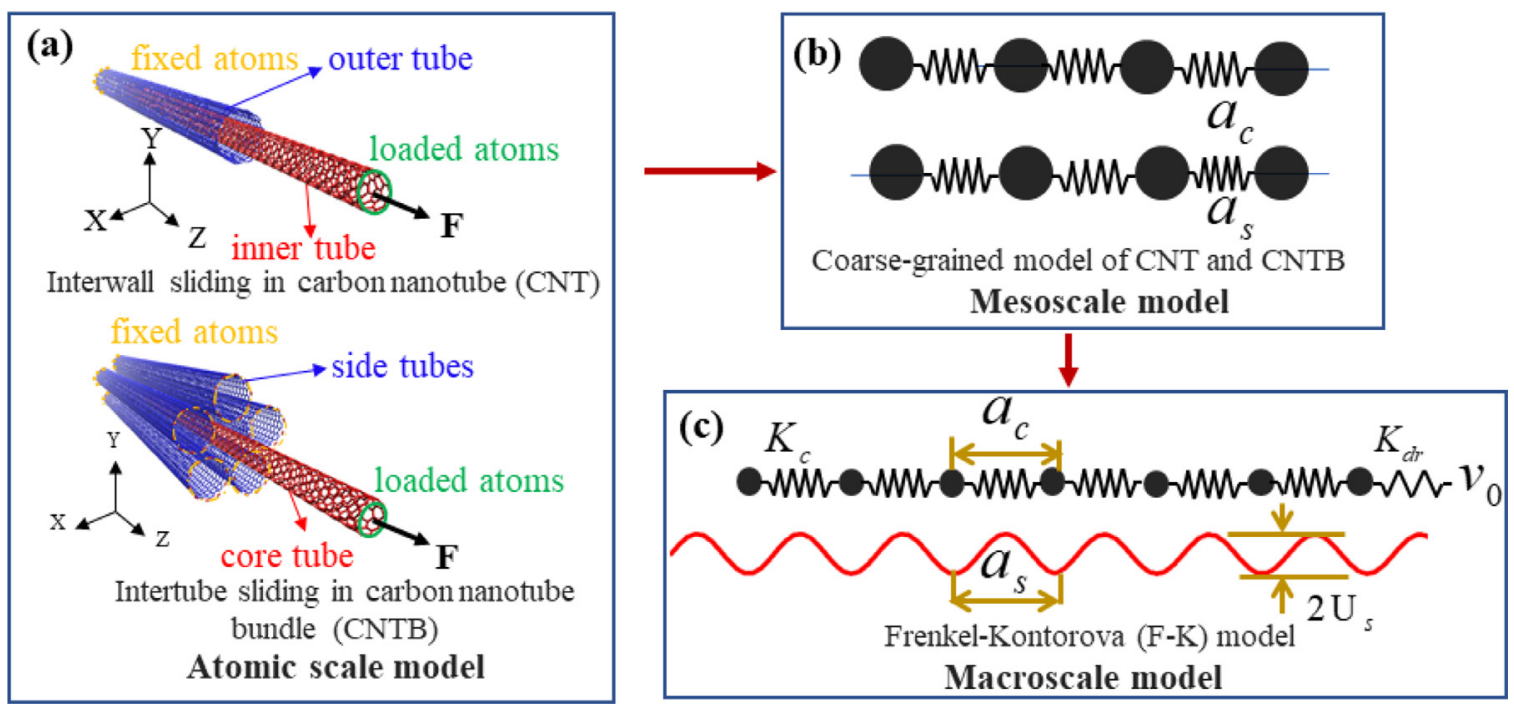

Fig. 5. The multi-scale model of CNT and CNTB. (a) illustrates the atomic scale models of inter-wall sliding in CNT and inter-tube sliding in CNTB. (b) shows the mesoscale model obtained by the coarse-grained method. (c) indicates the macroscale model, which corresponds to the F-K model.

ignored. Due to the strong in-plane interaction, distance between adjacent atoms is hardly affected by the weak interlayer interaction. As a result, the individual carbon nanotube can be assumed as rigidity. Interaction between interlayer atoms can be equivalent to a sinusoidal potential function and this equivalence corresponds to the Frenkel-Kontorova (F-K) model (Braun and Kivshar, 2004), which extends the computation model to macroscale (Fig. 5c).

\subsection{The atomic scale model of $C N T / C N T B$}

To obtain the basic parameters of carbon nanotube and the inter-wall/ inter-tube interaction, a series of MD simulations are carried out using an open sources LAMMPS (Large-scale Atomic/Molecular Massively Parallel Simulator) (Plimpton, 1995). In this study, the Adaptive Intermolecular Reactive Empirical Bond Order (AIREBO) potential (Stuart et al., 2000), which is able to simulate the bond formation and bond breaking of $\mathrm{C}-\mathrm{C}$, is adopted to describe the $\mathrm{C}-\mathrm{C}$ bonded interaction. The Lenard-Jones (LJ) 12-6 potential (Halgren, 1992) given by Eq. (1) is used to describe the long-range vdW non-bonded interactions between carbon atoms, and the cut-off distance is set as $12 \AA$ (Yuan and Wang, 2017; Yuan and Wang, 2018). For carbon atoms, the depth of the potential well $\varepsilon_{\mathrm{C}-\mathrm{C}}$ and the zero-potential distance $\sigma_{\mathrm{C}-\mathrm{C}}$ are set as $0.00284 \mathrm{eV}$ and $3.4 \AA$ (Yuan and Wang, 2017; Yuan and Wang, 2018), respectively. To be consistent with the experimental environment, the NVT (constant Number of atoms, Volume and Temperature) ensemble is adopted and the temperature is controlled around 300 K using the Nosé-Hoover thermostat (Nosé, 1984). The Verlet algorithm is employed to perform the time integration with a time step of $0.001 \mathrm{ps}$.

$$
E=4 \varepsilon_{C-C}\left[\left(\frac{\sigma_{C-C}}{r}\right)^{12}-\left(\frac{\sigma_{C-C}}{r}\right)^{6}\right]
$$

Firstly, the atomic model of individual CNT is established, which is based on the fact that CNT is formed by curling the graphene sheet. The curling process is shown in Fig. 6(a) and the coordinate transformation relation is expressed in Eq. (2). Moreover, the different geometries of CNT, depending on the way that the graphene sheet is curled, are specified by the integers $(n, m)$ and the diameter of the nanotube is given by Eq. (3). Then, the so-called armchair nanotubes correspond to the integers $(n, n)$, the zigzag ones to $(n, 0)$, and the chiral nanotubes to $(n, m)$. Due to the fact that most of the DWCNTs in our experiments, especially those (Zhang et al., 2013a), have the incommensurate configurations, the ultra-low interwall interaction is measured (Zhang et al., 2013a). Then, simulations are carried out in the $(9,0) @(10,10)$ system. The initial length of the nanotube $(9,0)$ is $199.86 \AA$ and it is $199.36 \AA$ for the nanotube $(10,10)$. Moreover, the effect of the commensurability is also discussed in the supporting information (Note S2).

$$
\begin{aligned}
& x_{P}=\frac{d}{2 \pi}-\frac{d}{2 \pi} \cos \frac{2 \pi y_{P^{\prime}}^{\prime}}{d}, y_{P}=\frac{d}{2 \pi} \sin \frac{2 \pi y_{P^{\prime}}^{\prime}}{d}, z_{P}=z_{P^{\prime}}^{\prime} \\
& d=\frac{\sqrt{3} a_{C-C}}{\pi} \sqrt{n^{2}+m^{2}+m n}
\end{aligned}
$$

wherea $a_{C-C}$ is the bond length of $\mathrm{C}-\mathrm{C}$. 

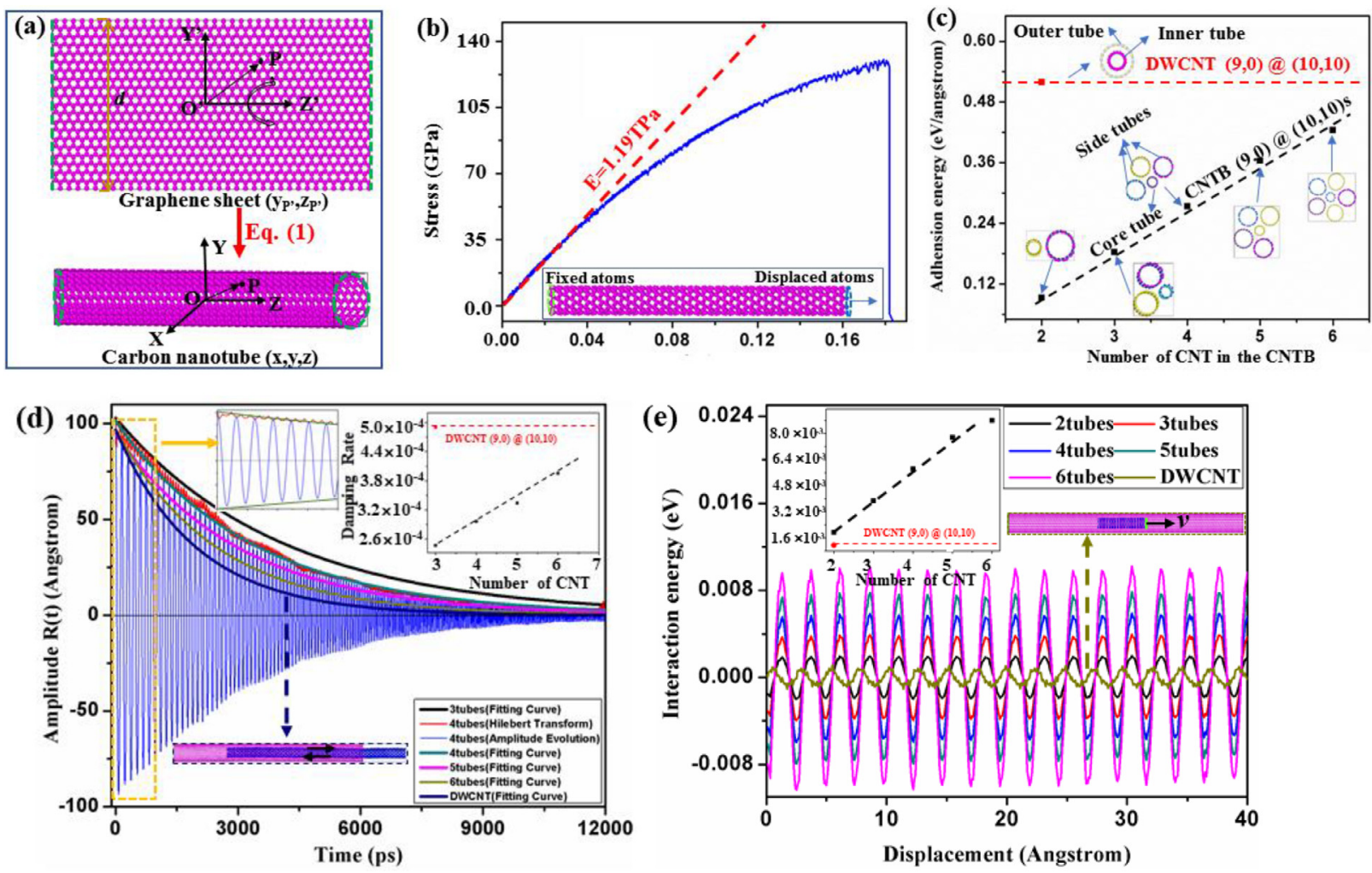

Fig. 6. The basic parameters of CNT/CNTB obtained by MD simulation. (a) illustrates the establishment of the individual CNT by curving the graphene. (b) is the stress-strain curve in the uniaxial tension test of CNT $(9,0)$ used to determine the Young's modulus. (c) shows the adhesion energy of inter-wall for DWCNT and inter-tube for CNTB calculated to obtained the updated LJ potential in the coarse-grained model. (d) expresses the amplitude attenuation process of the inner/core tube with time, where the dynamic friction coefficient can be obtained by the coast-down test. (e) shows the variation of the interaction energy when a short tube moves relatively to long tubes, where the parameters of the interaction energy can be obtained without considering the boundary.

Then, to determine the basic parameters of carbon nanotube and the inter-wall/inter-tube interaction, four mechanical loading cases are conducted.

The first one is the tensile loading case, which is used to determine the Young's modulus of the pulled-out tube $(9,0)$. After the initial structure is relaxed to reach the equilibrium state with minimum energy, the tube is stretched slowly at one end with the other end fixed. Based on the MD calculation, the tensile curve is obtained and shown in Fig. 6(b). The Young's modulus can be expressed as the slope of this stress-strain curve at the initial stage, which is 1.19TPa.

The second loading case is an assembly of CNTs, including $(9,0) @(10,10)$ for DWCNT and $(9,0) @(10,10)$ s for CNTB, which is conducted to obtain the adhesion energy. For the DWCNT, with one end of the inner tube $(9,0)$ and the opposite end of the outer tubes $(10,10)$ fixed, the system is relaxed to reach the minimum energy. For the CNTB, however, the system is relaxed when one end of the core tube $(9,0)$ is fixed and the detailed description of the establishing process is shown in the supporting information (Note S3). Then, the minimum vdW interaction energy of the inter-wall/inter-tube is calculated and shown in Fig. 6(c). It can be found that, with the CNT number increasing, the adhesion energy per unit length of CNTB increases linearly and tends to that of DWCNT. This can attribute to the increasing contact area between CNTB with the number of CNT increasing, while it is the maximum for DWCNT. The configurations of the DWCNT and CNTB are described in Fig. 7(a).

The third one is the coast-down test (Servantie and Gaspard, 2006b; Cook et al., 2013), which is carried out to obtain the dynamic friction coefficient. With the inner/core tube given an initial extraction of $100 \AA$, the system is relaxed to the equilibrium state. Then, the inner/core tube will oscillate along the axial due to vdW interaction after the fixed side is released. Taking the CNTB with four tubes for example, as shown in Fig. 6(d), the core tube oscillates along the axial and the maximum amplitude of this mechanical oscillation will decay due to the energy dissipation. To quantitatively describe the amplitude evolution with time, the Hilbert transformation (Gabor, 1946) is applied to obtain the envelope of the oscillation curve. According to the envelope curve, the attenuation process can be described by Eq. (4) through least square fitting (Servantie and Gaspard, 2006a; Servantie and Gaspard, 2006b). The mechanism of the energy dissipation is the generation of phonons in this process. Atoms in this test experience the proper physics, including breathing and twisting acoustic modes, so phonons will be excited by the interaction between walls/tubes (Cook et al., 2013; Prasad and Bhattacharya, 2017; 

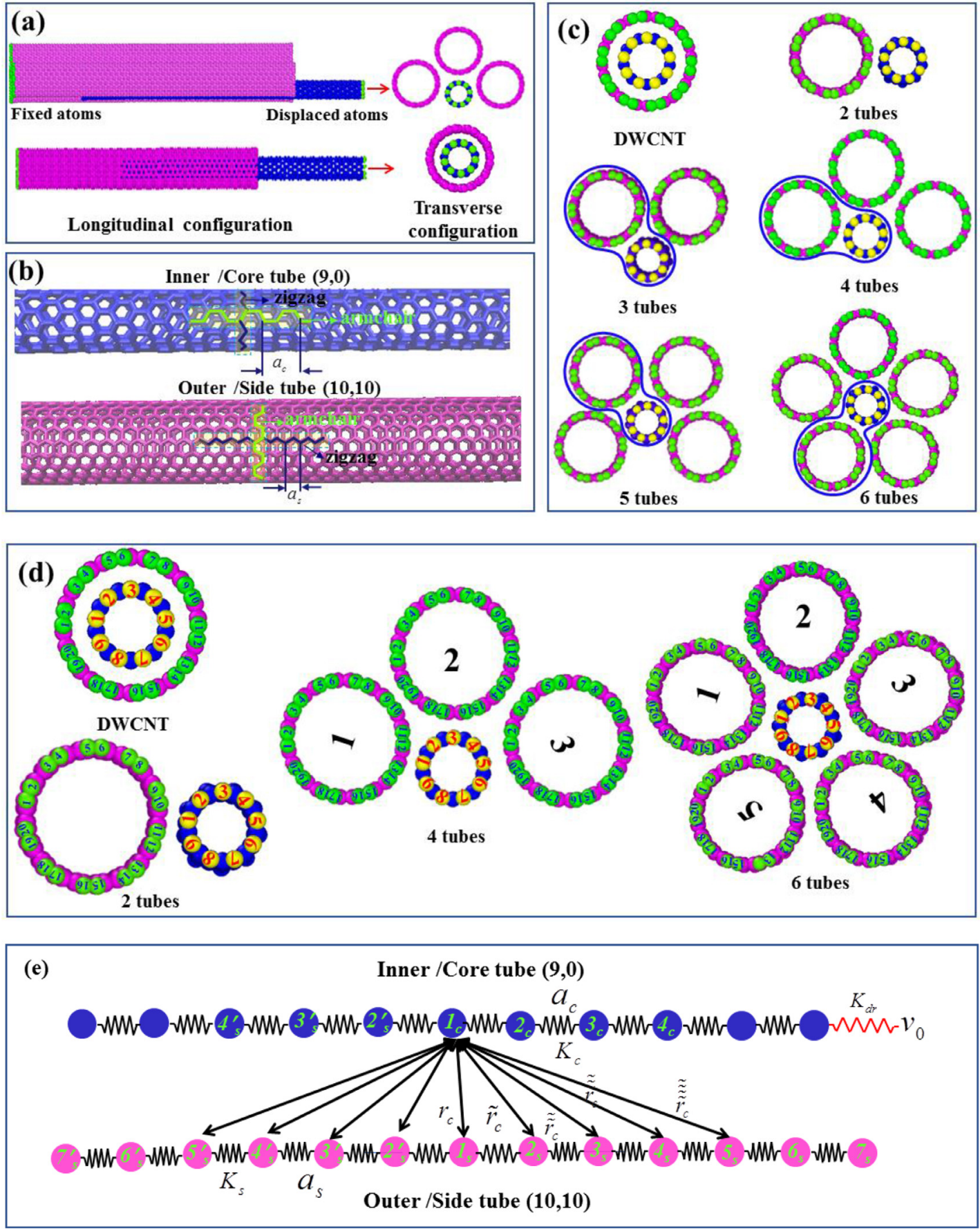

Fig. 7. The modeling process from atomic scale to mesoscale based on the coarse-grained method. (a) shows configuration of the DWCNT and the CNTB with 4 tubes. (b) is the detailed configuration of the DWCNT and the CNTB along axial directions. (c) expresses the circumferential configuration of the DWCNT/CNTBs. (d) shows the detailed configuration of the selected DWCNT/CNTB along the circumferential directions. (e) illustrates mesoscale model based on the coarse-grained method.

Zhang et al., 2003; Hepplestone and Srivastava, 2006). Then, the phonon dissipation results in the accumulation of thermal energy and the system heats up. However, to keep the temperature of the system constant, the thermostat on the outer/side tube continually extracts heat, resulting in the consistent with the experimental environment.

$$
R(t)=R_{0} \exp \left(-\Gamma_{R} t\right)=R_{0} \exp \left(-\frac{2 \gamma}{3 \mu} t\right)
$$


where $R(t)$ is the maximum amplitude of this mechanical oscillation, $\Gamma_{R}$ is the damping rate, $\gamma$ is the dynamic friction coefficient and $\mu=\left(N_{1} N_{2}\right) /\left(N_{1}+N_{2}\right) m$ is the relative mass of the system. Here, $N_{1}$ and $N_{2}$ are the number of atoms in inner/core and outer/side tubes, and $m=12$ amuis the atomic mass of carbon. Moreover, with the number of the side tubes increasing, the damping rate increases linearly for the CNTB, as illustrated in the small graph of Fig. 6(d). This is because that the contact area multiplied, the phonon dissipation shows great growth. Thus, the damping rate is maximum for the DWCNT because this system has the largest contact area.

The forth loading case is conducted to determine the parameters of interaction energy without considering the boundary, where a short tube $(9,0)(40 \AA)$ moves relatively to long tubes $(10,10)$ s $(199.36 \AA)$. After the system is relaxed to the minimum energy, the short tube is pulled forward along the axial at constant speed $(0.1 \AA / p s)$. Then, the potential energy of the inter-wall/inter-tube interaction is calculated in Fig. 6(e), where the constant term is subtracted to make comparison because the contribution to the pull-out force from this part is the derivative of the interaction energy with displacement and is independent of the constant term. The interaction energy in this loading case presents sinusoidal variation and the amplitude increases linearly with the CNT number multiplied for the CNTB. This attributes to the appearance of the commensurate configuration. To further illustrate the phenomenon, the detailed configurations of the DWCNT and CNTBs along axial and circumferential directions are described in Fig. 7(b) and (c). For the DWCNT, it is incommensurate along any direction because the chirality of the inner tube is armchair along axial direction and zigzag in circumferential direction, while the chirality of the outer tube is reversed. However, for the CNTBs, they are incommensurate along the axial direction, where the chirality of the core tube $(9,0)$ is armchair and the side tubes are zigzag. For the circumferential configurations, the CNTBs are commensurate. This attributes to the fact that the side tube can freely rotate and translate around the core tube in the relaxation, resulting in the circumferential configuration superposition for CNTBs with the number of the side tubes increasing. The circumferential configuration of the CNTB with 2 tubes after relaxation is shown in Fig. 7(d), where the atom 1 of the core tube locates between the atoms 10 and 11 of the side tube. In this situation, the atom 1 of the core tube falls at the bottom of potential well formed by the atoms 10 and 11 of the side tube, resulting in the maximum interaction energy. Then, taking the CNTB with 4 tubes as an example, the circumferential configuration is the superposition of three CNTBs with 2 tubes, where the atoms 1, 3 and 5 of the core tube lay between atoms 12 and 13 of side tube 1, atoms 16 and 17 of side tube 2 and atoms 20 and 1 of side tube 3, respectively. Therefore, the circumferential configurations of the CNTBs are commensurate and the amplitude of the interaction energy satisfies linear superposition in Fig. 6(e). However, the CNTB with 6 tubes is a special case. It can be fund that only the configuration formed by the core tube and the side tube 5 is the same as that of the CNTB with 2 tubes, where the atom 8 of the core tube locates between the atoms 6 and 7 of the side tube 5. But the configurations composed with the core tube and the side tubes $1,2,3$ and 4 are not the same as that of the CNTB with 2 tubes. Thus, the CNTB with 6 tubes is not exactly the superposition of five CNTBs with 2 tubes and the amplitude of the interaction energy no longer satisfies the linear superposition. As a result, the amplitude of the interaction energy is then below the line in Fig. 6(e).

\subsection{The mesoscale model of $C N T / C N T B$}

Based on the coarse-grained method, the mesoscale model of the CNT/CNTB is established. For the incommensurate system shown in Fig. $7(\mathrm{a})$, the chirality of the outer/side tube $(10,10)$ is zigzag along axial and armchair in circumferential direction, while it is opposite for inner/core tube $(9,0)$. Thus, the period of the outer/side tube $(10,10)$ is $a_{s}=\sqrt{3} a_{c-c}=2.46 \AA$ and $a_{c}=3 a_{c-c}=4.26 \AA$ for the inner/core tube $(9,0)$ along axial, respectively. In the mesoscale model shown in Fig. 7(b), the atomistic representation of the CNT is replaced by a collection of beads interacting with various molecular potentials. The $\mathrm{C}-\mathrm{C}$ bonded interaction is replaced by the harmonic spring and the long-range vdW non-bonded interaction is represented by the updated Lenard-Jones (LJ) 12-6 potential.

According to the period of the outer/side and inner/core tubes, the mass of a single particle shown in Fig. 3(b) is given $\operatorname{bym}_{(9,0)}^{a_{c}}=432 a m u a n d m_{(10,10)}^{a_{s}}=480 a m u$. The spring constants calculated from Young's modulus for small deformation can be expressed as:

$$
\begin{aligned}
& K_{c}^{(9,0)}=F / \Delta x=\sigma_{x} \times A_{(9,0)} /\left(\varepsilon_{x} \times a_{c}\right)=A_{(9,0)} \times E / a_{c} \\
& K_{s}^{(10,10)}=F / \Delta x=\sigma_{x} \times A_{(10,10)} /\left(\varepsilon_{x} \times a_{s}\right)=A_{(10,10)} \times E / a_{s}
\end{aligned}
$$

where $F, \Delta x, \sigma_{x}, \varepsilon_{x}$ andAare tensile loading, elongation, stress, strain and cross-sectional area of the tube. Thus, the spring constants are $K_{c}^{(9,0)}=6.57 \times 10^{5} \mathrm{amu} / \mathrm{ps}^{2}$ and $K_{s}^{(10,10)}=42.14 \times 10^{5} \mathrm{amu} / \mathrm{ps}^{2}$. For the updated Lenard-Jones (LJ) 12-6 potential, the updated potential well $\varepsilon_{C-C}$ and the zero-potential distance $\sigma_{C-C}$ should be determined. Because the equilibrium distance between inner/core and outer/side tube is $r_{c}=3.4 \AA$, the minimum interaction energy will be obtained in Eq. (1) when $r=$ $r_{c}$. Therefore, the updated zero-potential distance $\sigma_{c-c}$ can be obtained $\sigma_{C-c}=r_{c} / \sqrt[6]{2}=3.03 \AA$. The updated potential well $\varepsilon_{c-c}$ is chosen based on the fact that the mesoscopic and the atomistic model feature the same adhesion energy per unit length. Due to the cut-off distance set as $12 \AA$ in atomic simulation, the interaction length of the outer/side tube to the inner/core particle $\left(1_{c}\right)$ along axial direction is $11.51 \AA$, including 9 particles $\left(1_{s}, 2_{s}, 3_{s}, 4_{s}, 5_{s}, 2^{\prime} s, 3_{s}^{\prime}, 4_{s}^{\prime}\right.$ and $\left.5_{s}^{\prime}\right)$ shown in Fig. 7(b). As a result, the adhesion energy per unit length of the mesoscopic model $E_{L}$ can be shown as:

$$
E_{L}=\frac{1}{a_{c}} \times\left[E\left(r_{c}\right)+2 E\left(\tilde{r}_{c}\right)+2 E\left(\tilde{\tilde{r}}_{c}\right)+2 E\left(\tilde{\tilde{r}}_{c}\right)+2 E\left(\tilde{\tilde{\tilde{r}}}_{c}\right)\right]
$$



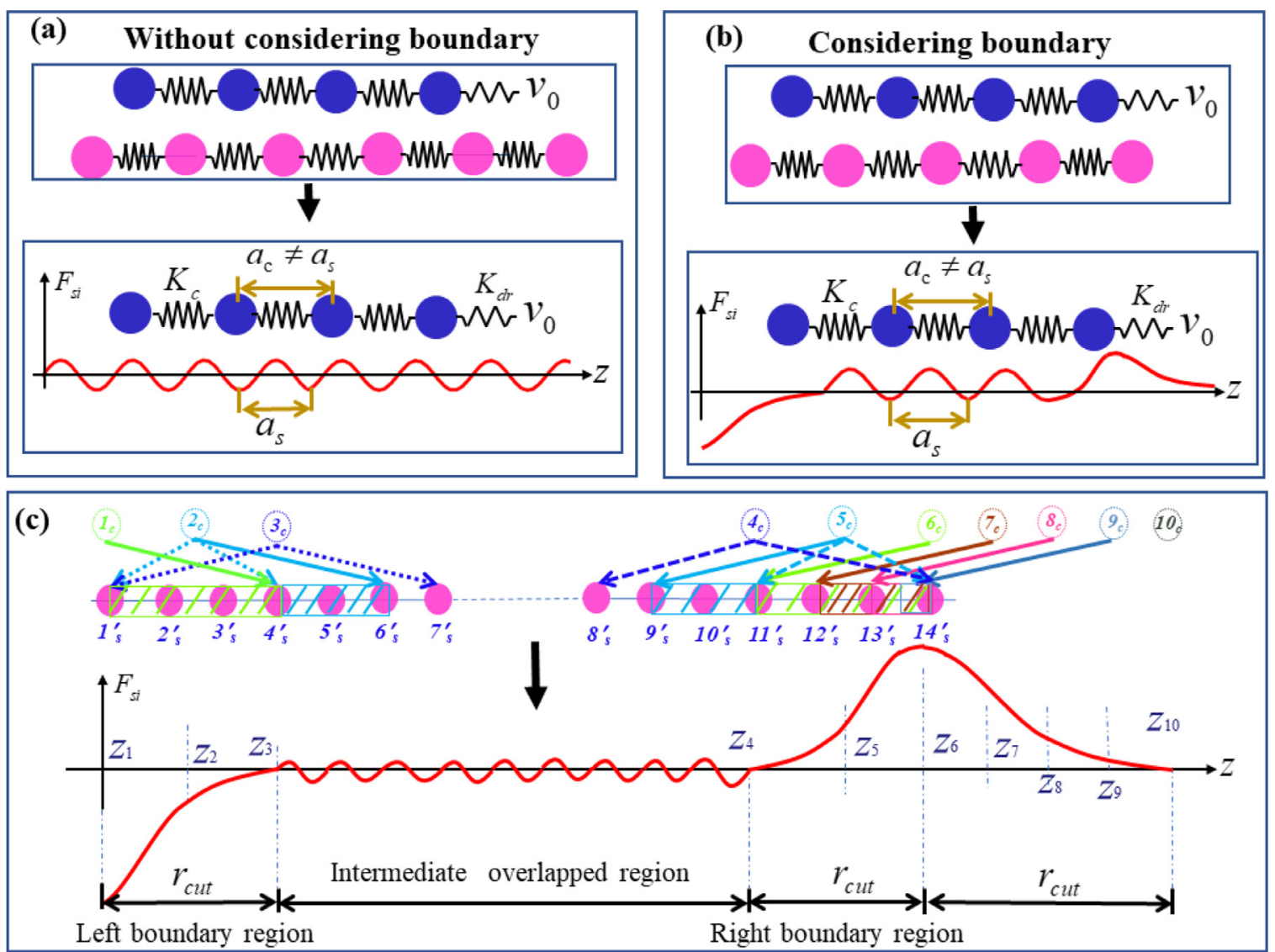

Fig. 8. The modeling process from mesoscale to macroscale of CNT/CNTB in the framework of Frenkel-Kotorova. (a) shows the modeling process without considering boundary, where the interaction force is equivalent to a sinusoidal function. (b) is the modeling process considering boundary, where the interaction force is quite complex at the boundary. (c) expresses the process when a particle, representing a part of the inner/core tube, moves relatively to the outer/side tube to obtain the corresponding interaction force.

Here,E(r)is the Lenard-Jones (LJ) 12-6 potential shown in Eq. (1) and the updated potential well $\varepsilon_{C-c}$ equalsE $\left(r_{c}\right)$.Therefore, according to Fig. $6(\mathrm{c})$, the updated potential wells $\varepsilon_{\mathrm{C}-\mathrm{c}}$ are $0.20 \mathrm{eV}, 0.40 \mathrm{eV}, 0.60 \mathrm{eV}, 0.80 \mathrm{eV}, 1.01 \mathrm{eV}$ and $1.06 \mathrm{eV}$ for 2 tubes, 3 tubes, 4 tubes, 5 tubes, 6 tubes in CNTB and DWCNT, respectively.

\subsection{The macroscale model of $C N T / C N T B$}

Although the mesoscale model can extend the computation scale to hundreds of nanometers in LAMMPS, the length of the ultra-long CNT /CNTB is usually several millimeters and the time scale is hundreds of seconds in the realistic experiment. To capture the main features of this huge and complex system, the macroscale model of the CNT/CNTB is developed to describe this dynamic problem phenomenologically. In order to be more consistent with the experimental process, the pull-out behavior of CNT/CNTB is analyzed in the framework of the Frenkel-Kotorova model. Compared to the strong covalent bond interaction in-plane, the weak inter-wall/inter-tube interaction can hardly affect the distance between adjacent atoms in the out/side tube. According to Benassi's report (Benassi et al., 2015), although the length of the CNT/CNTB in the experiment is several millimeters, the critical length, where the commensurate configuration appears due to the deformation of the tube, is $0.5 \mathrm{~m}$. Thus, the non-bonded vdW interaction can be equivalent to a potential function. Therefore, as shown in Fig. 8, the experimental process can be treated as chains of $N$ particles of massm ${ }_{(9,0)}^{a_{c}}$, connected by springs of stiffness $K_{c}^{(9,0)}$, having rest length $a_{c}$, and driven by a spring of constant $K_{d r}$ meaning the lateral stiffness of the probe cantilever, with speed of $v_{0}$ on a potential with periodicity representing the interaction with the outer/side tube.

When the inner/core tube is pulled out from the outer/side one, four parts of energy are included in this system shown in Eq. (7a), consisting of the elastic potential energy of the springs $U_{p}$, the kinetic energy of the particles $T_{p}$, the interaction energy with the outer/side tube $U_{s}$ and the dissipation energy caused by phonon dissipation $\Psi_{s}$. Meanwhile, to make the calculation simpler, a dimensionless unit system is introduced by setting $a_{c}$ as the length unit, $\tau=\sqrt{m_{(9,0)}^{a_{c}} / K_{c}^{(9,0)}}$ as the time unit and $U=K_{c}^{(9,0)} \times a_{c}^{2}$ as the energy unit. Then, according to the generalized Lagrange equation shown in Eq. (7b), the 
movement equations of particles can be obtained.

$$
\begin{aligned}
& u_{p}=\frac{U_{p}}{U}=\frac{1}{2} \sum_{i=2}^{N}\left(u_{i}-u_{i-1}\right)^{2} \\
& t_{p}=\frac{T_{p}}{U}=\frac{1}{2} \sum_{i=1}^{N}\left(\frac{d u_{i}}{d t^{\prime}}\right)^{2} \\
& u_{s}=\frac{U_{s}}{U}=-\frac{U_{0}}{K_{c}^{(9,0)} \times a_{c}^{2}} \sum_{i=1}^{N} \cos \left(2 \pi \times \frac{\left[u_{i}+(i-1)\right]}{a_{s} / a_{c}}\right)^{2} \\
& \varphi_{s}=\frac{\Psi_{s}}{U}=\frac{1}{2} \times \frac{\gamma}{m_{(9,0)}^{a_{c}}} \times \sum_{i=1}^{N}\left(\frac{d u_{i}}{d t^{\prime}}\right)^{2} \\
& \frac{d}{d t^{\prime}}\left(\frac{\partial t_{p}}{\partial \dot{u}_{i}}\right)+\frac{\partial\left(u_{p}+u_{s}\right)}{\partial u_{i}}+\frac{\partial \varphi_{s}}{\partial \dot{u}_{i}}=f_{i}
\end{aligned}
$$

Here, $t_{p}=T_{p} / U, u_{p}=U_{p} / U, u_{s}=U_{s} / U$ and $\varphi_{s}=\Psi_{s} /$ Uare the corresponding dimensionless energy parameters. $t^{\prime}=t / \tau, \dot{u}_{i}=\dot{U}_{i} \times \tau / a_{c}, u_{i}=U_{i} / a_{c}$ and $f_{i}=F_{i} /\left(K_{c} \times a_{c}\right)$ are the dimensionless parameters of time, speed, displacement and external loading. $U_{0}$ is the amplitude of the cosine potential function.

Here, the interaction force $\left(F_{s i}=\partial u_{s} / \partial u_{i}\right)$ obtained based on Eq. (7a) is considered when the boundary is taken into consideration because it is rather different compared to the condition without considering that. As shown in Fig. 8(a), the interaction force is a sinusoidal function without considering the boundary. The period and amplitude of this function can be obtained from Fig. 6(e) and Eq. (7a). However, the interaction force, as shown in Fig. 8(b), is no longer the sinusoidal function when particles are on the boundary. To clearly clarify the different, the change of the interaction force when a particle representing part of the inner/core tube is driven on the out/side tube is shown in Fig. 8(c), where the cut-off distance is set as $11.51 \AA$ along $\mathrm{z}$ axis as discussed in Section 3.3. At the left boundary, when the particle is located at $1_{c}$, particles $1_{s}^{\prime}, 2^{\prime}{ }_{s}, 3^{\prime}{ }_{s}$ and $4^{\prime}{ }_{s}$ act rightward force on it, resulting in the maximum resultant force. Then, as the particle moves to $2_{c}$, the resultant rightward force decreases because it is dragged leftward by particles $1^{\prime}{ }_{s}$ and $2^{\prime}{ }_{s}$. With the particle moving to $3_{c}$, the rightward and leftward forces at the boundary cancel out, resulting in the same force function as that without considering the boundary. However, when moving to the right boundary, the interaction force for the particle at $4_{c}$, $5_{c}$ and $6_{c}$ is the same as that at $3_{c}, 2_{c}$ and $1_{c}$ except for the opposite direction. With the particle moving out of the outer/side tube, corresponding to the positions of $7_{c}, 8_{c}, 9_{c}$ and $10_{c}$, the leftward force decreases to zero. As discussed above, the analytic interaction force $F_{s i}$ can be deduced in Eq. (8).

Without considering boundary:

$$
F_{s i}=\frac{U_{0}}{K_{c} a_{c}^{2}} \times \frac{2 \pi}{a_{s} / a_{c}} \sin \left[2 \pi \times \frac{u_{i}+(i-1)}{a_{s} / a_{c}}\right]
$$

Considering boundary:

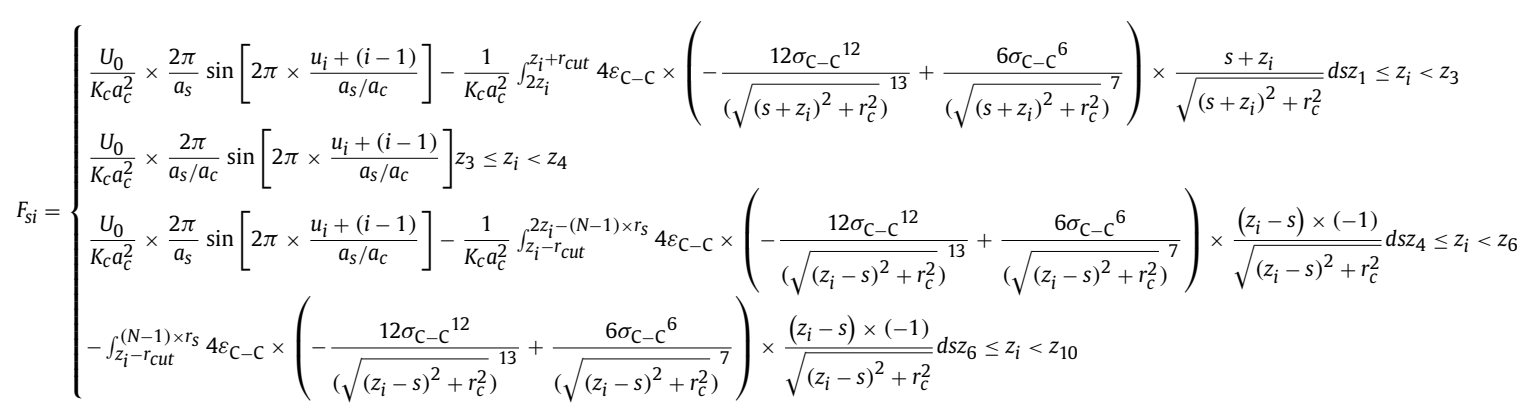

where $F_{s i}$ is the analytic interaction force of particle $i$, and $z_{i}$ is the axial coordinate of particle.

\section{Results and discussion}

\subsection{Comparison with the MD simulation of the pull-out process}

To validate the multi-scale model, the process of pulling the inner tube from the outer one in DWCNT is calculated by setting the parameters according to the atomic model in the MD simulation. Results obtained by the multi-scale model and the MD simulation are then compared. 
Table 1

Parameters of the multi-scale model according to the atomic model in the MD simulation.

\begin{tabular}{ll}
\hline Parameters & Magnitude \\
\hline Number of particles $\left(N_{1}\right)$ & 47 \\
Initial spacing between particles $\left(a_{c 1}\right)$ & $4.26(\AA)$ \\
Mass of each particle $\left(m_{(9,0) 1}^{a_{c}}\right)$ & $432(\mathrm{amu})$ \\
Stiffness of springs between particles $\left(K_{c}^{(9,0)}{ }_{1}\right)$ & $6.57 \times 10^{5}\left(\mathrm{amu}^{2} / \mathrm{ps}^{2}\right)$ \\
Stiffness of the spring representing the probe $\left(K_{d r 1}\right)$ & $6.57 \times 10^{5}\left(\mathrm{amu}^{2} / \mathrm{ps}^{2}\right)$ \\
Amplitude of the potential function $\left(U_{01}\right)$ & $1.15 \times 10^{-3}(\mathrm{eV})$ \\
Period of interaction potential function $\left(a_{s 1}\right)$ & $2.46(\AA)$ \\
The dynamic friction coefficient of one particle $\left(\gamma_{1}\right)$ & $0.22(\mathrm{amu} / \mathrm{ps})$ \\
The pull-out speed $\left(\nu_{1}\right)$ & $0.01(\AA / \mathrm{ps})$ \\
The updated zero-potential distance $\left(\sigma_{\mathrm{c}-\mathrm{c} 1}\right)$ The updated potential well $\left(\varepsilon_{\mathrm{C}-\mathrm{C} 1}\right)$ & $3.03(\AA) 1.06(\mathrm{eV})$ \\
The cut-off distance along axial $\left(r_{c u t}\right)$ & $11.51(\AA)$ \\
\hline
\end{tabular}
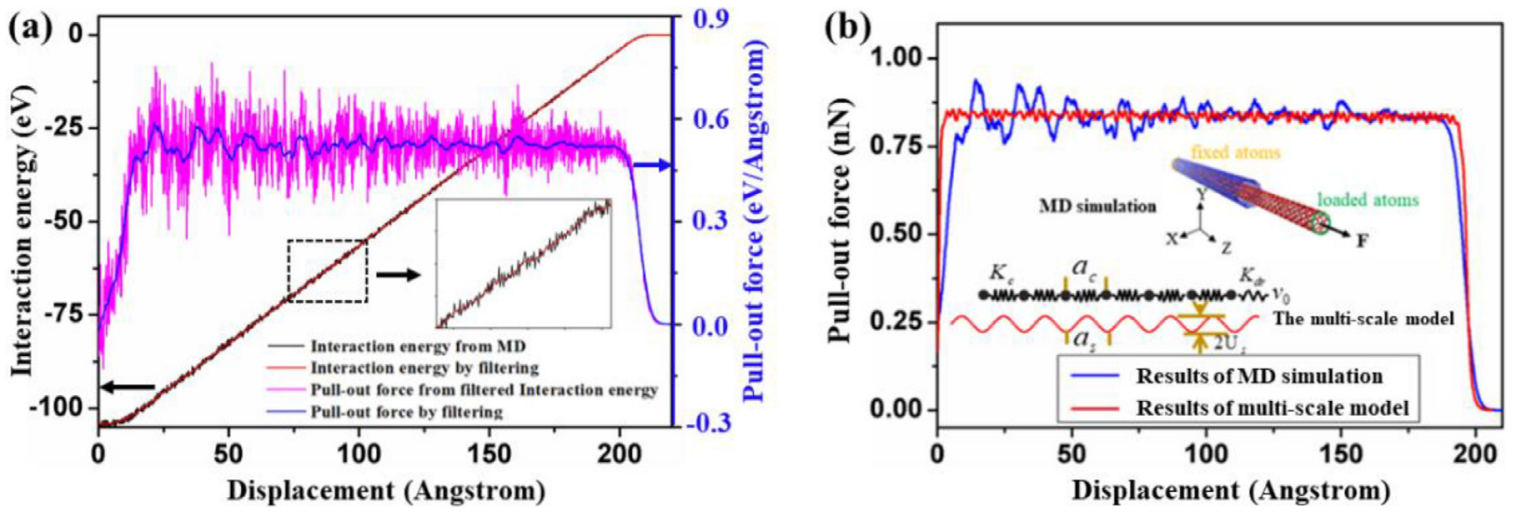

Fig. 9. Comparation of the pull-out process of the results of the MD simulation and multi-scale model. (a) shows the treatment of the interaction energy with much noise to obtain the pull-out force in the MD simulation. (b) compares the pull-out force variation of the MD simulation and multi-scale model with the inner tube being pulled out.

For the MD simulation, the computation system is the same as that in Section 3.1. The DWCNT of the (9,0) @ (10, 10) system is chosen, the initial length of the inner tube $(9,0)$ is $199.86 \AA$ and it is $199.36 \AA$ for the outer tube $(10,10)$, the parameters of LJ potential are $0.00284 \mathrm{eV}$ and $3.4 \AA$ for $\varepsilon_{\mathrm{C}-\mathrm{C}}$ and $\sigma_{\mathrm{C}-\mathrm{C}}$, the cut-off distance is $12 \AA$ and the pull-out velocity is $0.01 \AA / p s$.

According to the parameter selection process described in Section 3, the parameters of the multi-scale model in this calculation is shown in Table 1 and the subscript 1 of the symbols means parameters are selected in this table.

In the MD simulation, the pull-out force is not available directly because of the excessive noise when the temperature of

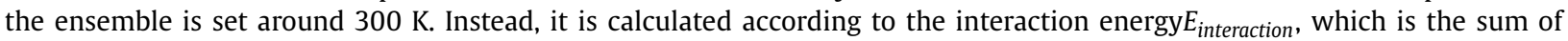
vdW interaction energy between inner and outer tubes shown in Eq. (9).

$$
E_{\text {interaction }}=\sum_{i=1}^{N_{o}} \sum_{j=1}^{N_{c}} 4 \varepsilon_{C-C}\left[\left(\frac{\sigma_{C-C}}{r_{i j}}\right)^{12}-\left(\frac{\sigma_{C-C}}{r_{i j}}\right)^{6}\right]
$$

where $i$ denotes an atom in the inner shell, $j$ is an atom in the outer shell and Nc, No are the numbers of atoms in the inner and outer shells, respectively. Then, the pull-out force can be obtained by $F_{\text {pull-out }}=\frac{\partial E_{\text {interaction }}}{\partial z}$. As shown of the enlarged box in Fig. 9(a), the interaction energy calculated from MD simulation has much noise, resulting in the great fluctuation of the pull-out force. Then, the Savitzky-Golay filtering technique (Savitzky and Golay, 1964) based on the least square fit algorithm is applied, by which the pull-out force can be obtained. However, the obtained pull-out force still has a lot of noise. To compare with the results given by the multi-scale model, the Savitzky-Golay filtering technique is applied to the fluctuated pull-out force again.

Fig. 9(a) shows the process of obtaining the pull-out force in the MD simulation and the comparation of the pull-out force variation with the multi-scale model is drawn in Fig. 9(b). The evolution of the pull-out force is almost the same, including the sharp increase stage, the slight periodic up-and-down stage and the rapid decrease stage. The maximum pull-out force obtained from the multi-scale model is nearly $0.83 \mathrm{nN}$, which is quite accord with the MD result. However, the mechanism of the computed pull-out force is quite different. The pull-out force calculated from MD simulation only includes the vdW interaction, which only depends on the relative position of the atoms between inner and outer tubes. So, the calculated pull-out force is nearly the same as that obtained from MS (Molecular Statics) because the weak interfacial interaction cannot change the length of the covalent (C-C) bonds in-plane. However, the pull-out force acquired in the multi-scale model 
Table 2

Parameters of the FK model describing the intermediate overlapped area based on the experiment.

\begin{tabular}{ll}
\hline Parameters & Magnitude \\
\hline Number of particles $\left(N_{2}\right)$ & 900 \\
Initial spacing between particles $\left(a_{c 2}\right)$ & $1 \times 10^{5}(\AA)$ \\
Mass of each particle $\left(m_{(9,0) 2}^{a_{c}}\right)$ & $1.01 \times 10^{7}(\mathrm{amu})$ \\
Stiffness of springs between particles $\left(K_{c}^{(9,0)}{ }_{2}\right)$ & $22.78\left(\mathrm{amu} / \mathrm{ps}^{2}\right)$ \\
Stiffness of the spring representing the probe $\left(K_{d r 2}\right)$ & $22.78\left(\mathrm{amu} / \mathrm{ps}^{2}\right)$ \\
Amplitude of the potential function $\left(U_{02}\right)$ & $1.15 \times 10^{-3}(\mathrm{eV})$ \\
Period of interaction potential function $\left(a_{s 2}\right)$ & $0.58 \times 10^{5}(\AA)$ \\
Dynamic friction coefficient of one particle $\left(\gamma_{2}=0.29 \times \gamma_{c}, \gamma_{c}=2 \times \sqrt{\left.K_{c}^{(9,0)}{ }_{1} \times m_{(9,0) 1}^{a_{c}} \times a_{c 2} / a_{c 1}\right)}\right.$ & $2.28 \times 10^{8}(\mathrm{amu} / \mathrm{ps})$ \\
The pull-out speed $\left(v_{2}\right)$ & $10^{-8}(\AA / \mathrm{ps})$
\end{tabular}

includes two parts. One part originates from the dynamic friction force caused by the energy dissipation and the other one is the vdW interaction. According to the formula $F_{r}=N_{1} \times \gamma_{1} \times v_{1}$ obtained according to Eq. (7a), the computed maximum dynamic friction force $F_{r}$ is $1.69 \times 10^{-5} \mathrm{nN}$, which is nearly four orders of magnitude smaller than vdW interaction force. The contribution of the vdW interaction force includes two parts: boundary effect and contribution of the configuration force. The pull-out force comes from the boundary effect is nearly $0.83 \mathrm{nN}$. While, the pull-out force contributed by the intermediate overlapped area $F_{s}$ is $5.12 \times 10^{-3} \mathrm{nN}\left(F_{s}=\sum_{i=1}^{N} \frac{U_{01}}{K_{c}^{(9,0)}{ }_{1}^{2} a_{c 1}^{2}} \times \frac{2 \pi}{a_{s 1} / a_{c 1}} \sin \left[2 \pi \times \frac{u_{i}+(i-1)}{a_{s 1} / a_{c 1}}\right]\right)$, which is nearly two orders of magnitude smaller than that resulted from boundary. As a result, the maximum pull-out force values are nearly the same in the two models and the pull-out force originated from the vdW interaction is mainly contributed by the boundary. Thus, the conclusion that the force of pulling the inner tube from the outer one is independent on the intermediate overlapped area is further verified (Cumings and Zettl, 2000; Suekane and Nakayama, 2008) and the mechanism is clearer.

\subsection{Comparison with the experimental results of the pull-out process}

The CNT/CNTB used in the experiment is usually several millimeters, so it is called ultra-long CNT/CNTB in the previous study (Zhang et al., 2013b; Zhang et al., 2016), where the pull-out behavior is hardly described by the previous computation method due to the limitation of computing space and time. While, the developed multi-scale model, which can extend the computation scale to macroscale, can be used to describe the pull-out process observed in the experiment. In Zhang's experiment (Zhang et al., 2013a), two 9-mm-long inner tubes are pulled out of the ultra-long DWCNT, of which the outer tubers' diameters are $2.73 \mathrm{~nm}$ and $3.26 \mathrm{~nm}$, respectively. Then, the relationship between the pull-out force and the length of the overlapped area is investigated. In Zhang's another experiment (Zhang et al., 2016), to investigate the relationship between the pull-out force and the pull-out velocity, a 3-mm-long DWCNT with diameter of $2.67 \mathrm{~nm}$ is used in their work. In addition, the ultra-long DWCNT used in our experiment is $2 \mathrm{~mm}$ long with outer tube's diameter of $2.67 \mathrm{~nm}$. As a result, the parameters set in Table 2 is completely based on the experiment.

Firstly, to realize the numerical calculation at macroscale for the multi-scale model, larger coarse-grained particles are set in the intermediate overlapped area due to the conclusion obtained in Section 4.1. It is proved that the pull-out force originated from the vdW interaction contributed by the intermediate overlapped area is nearly two orders of magnitude smaller than that of the boundary effect, which is independent of the overlapped area length due to the incommensurate configuration.

Then, one key technique of the multi-scale model is to appropriately describe the junctions of the two boundary regions and the intermediate overlapped region. These three regions are shown in Fig. 8(c), including the left boundary region, the intermediate overlapped region and the right boundary one. Thus, the sketch chart of the computation process is drawn in Fig. 10 specially to solve the problem. Because the cut-off distance $\left(r_{\text {cut }}\right)$ is set as $11.51 \AA$ and the period of the particles at the boundary $\left(a_{c 1}\right)$ is $4.26 \AA$, in the initial state (State 1 ), three particles are located at the left and right boundary region, respectively. With the particle chain moving rightward, particles in the left boundary region will enter the right side of the intermediate overlapped region and the particle in the right side of the intermediate overlapped region will get into the right boundary region. When the displacement of the particle chain is greater than $2.49 \AA\left(r_{c u t}-2 a_{c} \geq 2.49\right)$, corresponding to State 2, particle $3 c$ vanishes in the intermediate overlapped region and reappears in the right boundary region for ease of understanding. Similarly, particle $2_{c}$ will experience the same process as that of particle $3_{c}$ when the displacement is larger than $6.75 \AA\left(r_{c u t}-a_{c} \geq 6.75\right)$. Then, when the displacement is over $11.51 \AA$ (State 4$)$, particles in the left boundary region all move into the intermediate overlapped region and the right-most particle $10_{c}$ reaches the edge of the right boundary region, beyond which the interaction force no long works. In State 5 , the right-most particle $10_{c}$ is out of the interaction range and new particle $10_{c}$ will add to this position until the displacement exceeds the period of the particles in the intermediate overlapped region $\left(a_{c 2}\right) 1 \times 10^{5} \AA$ (State 6), where one larger particle $\left(7_{c}\right)$ in the intermediate overlapped region escapes from the interwall interaction.

Here, another key technique is to determine the dynamic friction coefficient in the macroscale because it can be found the dynamic friction force based on the parameter in Table $1\left(\gamma_{1}=0.22 \mathrm{amu} / \mathrm{ps}\right)$ is $1.27 \times 10^{-5} \mathrm{nN}$ when the length of 


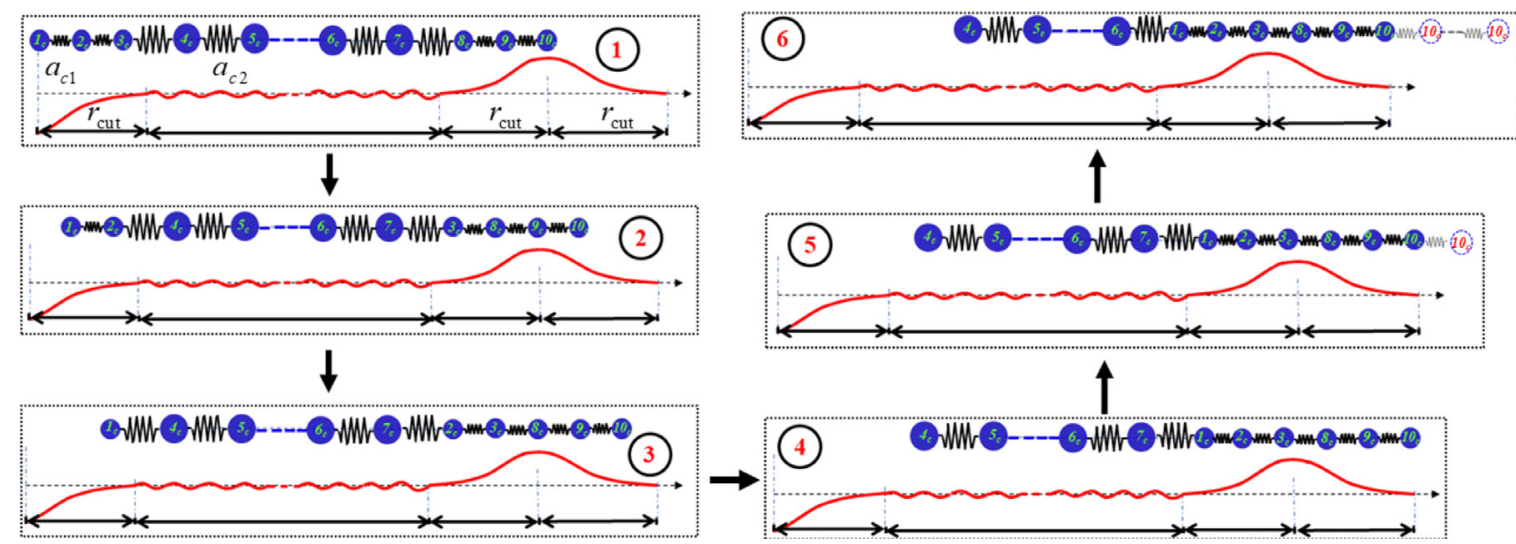

Fig. 10. The computation sketch chart of the junctions in the macroscale model, where the small particles $1 c, 2 c$ and $3 c$ are in the left boundary region, big particles $4 c, 5 c, 6 c$ and $7 c$ are in the intermediate overlapped region, and $8 c, 9 c$ and $10 c$ are in the right boundary region.
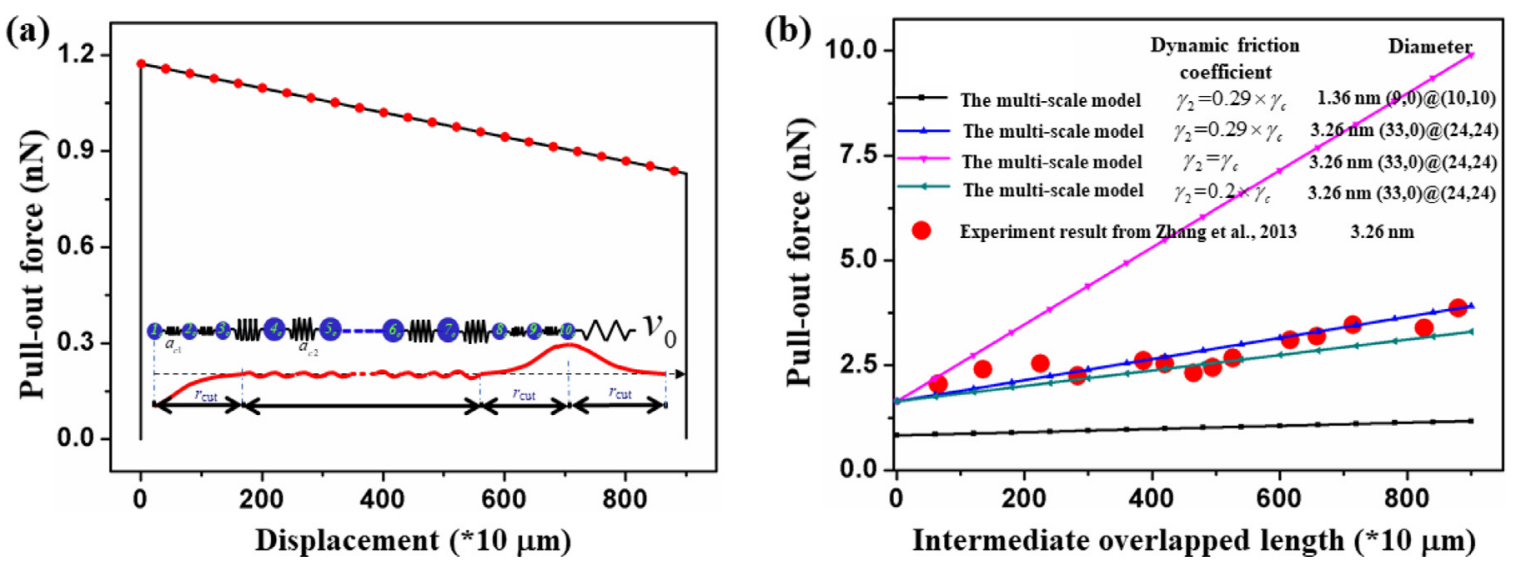

Fig. 11. Comparation of the pull-out process of the results from multi-scale model and experiment (Zhang et al., 2013a). (a) shows the pull-out force variation in the multi-scale model with the inner tube being pulled out, which is quite different that of the atomic scale model in Fig .9(a). (b) compared the results of the macroscale model with the experiment results to clarify the pull-out mechanism in the ultra-long DWCNT.

ultra-long DWCNT is $3 \mathrm{~mm}$ and pull-out speed is $5 \times 10^{-8} \AA / \mathrm{ps}(5 \mu \mathrm{m} / \mathrm{s})$, which is far less than the experimental value (4.2 $\mathrm{nN}$ ) measured by Zhang (Zhang et al., 2016) and shown in Fig. 12. This means the dynamic friction coefficient obtained by the MD simulation is not suitable for the macroscale, especially in the experiment. This mainly attributes to the mismatch of the time scale because the speed is $0.01 \AA / p s$ in the MD simulation and it is $10^{-8} \AA / p s$ in the experiment. Then, the previous studies of the friction forces at the atomic level using friction force microscopy (FFM) are referenced (Krylov et al., 2006; Krylov and Frenken, 2012), where the critical damping $\left(\gamma_{c}=2 \sqrt{m \times k}\right)$ is usually assumed. Here, $m$ and $k$ are the mass and stiffness of the probe, respectively. In addition, the pull-out process of the DWCNT is an underdamping motion based on the coast-down test shown in Fig. 6(d). As a result, twenty percent of the critical damping is generally assumed in engineering (Clough and Penzien, 2003). Based on the assumption $\left(\gamma_{2}=0.2 \times \gamma_{c}, \gamma_{c}=2 \times \sqrt{K_{c}^{(9,0)}{ }_{1} \times m_{(9,0) 1}^{a_{c}}} \times a_{c 2} / a_{c 1}\right)$, the dynamic friction force is $3.8 \mathrm{nN}$ when the parameters are set according to the experiment, which is comparable with but less than the experimental value $(4.2 \mathrm{nN})$ because of the smaller outer tube diameter $(1.36 \mathrm{~nm}$ for the outer tube (10, 10) in the multi-scale model). Then, the dynamic friction coefficient in the multi-scale model can be determined by adjusting the parameter $\alpha\left(\gamma_{2}=\alpha \times \gamma_{c}, \gamma_{c}=2 \times \sqrt{K_{c}^{(9,0)}{ }_{1} \times m_{(9,0) 1}^{a_{c}}} \times a_{c 2} / a_{c 1}\right)$ and setting the same diameter of the outer tube as that of the experimental sample. By matching the experimental data shown in Figs. 11 and 12, the parameter $\alpha$ equals 0.29.

Based on the considerations mentioned above, the parameters of the particles in the intermediate overlapped area are set in Table 2. Parameters of the particles at the boundary are the same as that in Table 1 . Subscript 2 of the symbols means parameters are selected in Table 2.

The pull-out process of the inner CNT from the DWCNT is calculated and compared with the experiment result in Fig. 11. Fig. 11(a) describes the pull-out process of the CNT based on the multi-scale model, which is quite different from that of the CNT on nanoscale as shown in Fig. 9(a). Instead of the slight periodic up-and-down motion, the pull-out force of CNT in the macroscale expresses a linearly decrease with the inner tube being pulled out. As described in Fig. 9(b), the pull-out force of 


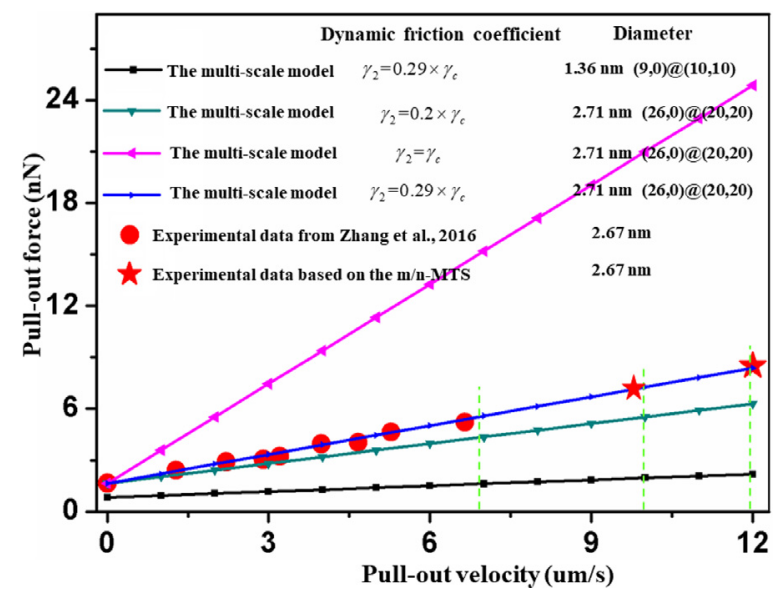

Fig. 12. Velocity effect on the pull-out force, including comparation of the results from multi-scale model and experiment, where the reference is Zhang et al., 2016.

the DWCNT on nanoscale is mainly contributed by the boundary, while dynamic friction force and the configuration force are too small to be ignored. However, although the contribution by the boundary effect keeps unchanged $(0.83 \mathrm{nN})$ for the ultralong DWCNT, the dynamic friction force increases significantly $(0.34 \mathrm{nN})$ because the larger overlapped area will result in more phonon dissipation, which is on the same order of the boundary contribution and cannot be ignored anymore. Therefore, the decreased pull-out force is attributed to the reducing interaction area. This mechanism can also be verified by the previous experiment result (Zhang et al., 2013a), as shown in Fig. 11(b), where the pull-out force shows linear increase with increasing the overlapped area. The curve slop representing the dynamic friction coefficient of the DWCNT in the experiment is larger than that of the computation system $((9,0) @(10,10))$. This can be easily obtained by the formula of the dynamic friction coefficient in Table 2. With the increase of the outer tube diameter, the mass of the particle and stiffness of the spring will increase at the same time, resulting in the larger dynamic friction coefficient of one particle. To compare with the experimental results, the $(33,0) @(24,24)$ system is computed by the multi-scale model, where the diameter of the outer tube is the same as that of the experimental sample $(3.26 \mathrm{~nm})$. By fitting the experimental data, the computation results are more reasonable when setting the parameter $\alpha$ as 0.29 in Table $2\left(\gamma_{2}=0.29 \times \gamma_{c}, \gamma_{c}=2 \times \sqrt{\left.K_{c}^{(9,0)}{ }_{1} \times m_{(9,0) 1}^{a_{c}} \times a_{c 2} / a_{c 1}\right)}\right.$. Moreover, the linear relationship between the pull-out force and the intermediate overlapped length cannot be obviously observed in our experiment (Fig. 3), which attributes to the small pulled out length of the inner tube (500 $\mu \mathrm{m})$.

The effects of the pull-out velocity are discussed in Fig. 12. The pull-out force shows nearly the linear relationship with the velocity acting on the inner tube from experimental result (Zhang et al., 2016). Our experimental data lies on the extension line of the experiment from reference. Thus, the linear relationship between the pull-out force and the acting velocity is verified further. The mechanism of this linear relationship can also be explained by the developed multi-scale model. As described in Fig. 11, the dynamic friction force cannot be ignored for the ultra-long DWCNT, which is related to the pull-out force and can be expressed as $f_{r}=N \times \gamma \times v$. This dynamic friction force is thus proved to be a Stokes-like (Müser, 2002) viscous drag, resulting in the linear relationship of the pull-out force and the velocity in our computation system. Moreover, because the diameter of the outer tube in the multi-scale model is smaller than that in the experiment, the slope of the curve, representing the dynamic friction coefficient $\gamma$, is less. To compare with the experiment result, the system $(26,0) @$ $(20,20)$, where the diameter of the outer tube is nearly the same as that of the experimental sample, is calculated based on the multi-scale model. Results from the multi-scale model are consistent with the experimental data when the parameter $\alpha$ is set as 0.29 Table $2\left(\gamma_{2}=0.29 \times \gamma_{c}, \gamma_{c}=2 \times \sqrt{K_{c}^{(9,0)}{ }_{1} \times m_{(9,0) 1}^{a_{c}}} \times a_{c 2} / a_{c 1}\right)$. Thus, this conclusion is further verified in Fig. 12 . However, for all we know, this linear relationship between the pull-out force and the velocity is not observed in the MD simulation in the previous study. As shown in Section 4.1, the dynamic friction force is four orders of magnitude smaller than the contribution from the boundary effects, so the increased pull-out force due to the increasing pull-out velocity will submerge in the noise. While, if the pull-out velocity is high enough for the pull-out force reaching the same orders of magnitude as the boundary effect, the inner tube will break instead of being pulled out. Moreover, as discussed in Section 2, the dynamic force is not manifested in the previous experiment test because of the low pull-out velocity and short DWCNT, for example, $3.1 \mathrm{nN}$ (length: $700 \mathrm{~nm}$; magnitude of pull-out velocity: $\mathrm{nm} / \mathrm{s}$ ) (Kis et al., 2006) and $4.0 \mathrm{nN}$ (length: $150 \mathrm{~nm}$; magnitude of pull-out velocity: nm/s) (Akita and Nakayama, 2003b).

\subsection{Prediction of the pull-out process of ultra-long CNTB}

Based on the multi-scale model proposed above, the pull-out behaviors of ultra-long CNTB can be predicted further. In the experiment, the ultra-long CNTB consists of three CNTs with diameters of $2.61 \mathrm{~nm}, 1.82 \mathrm{~nm}$ and $2.02 \mathrm{~nm}$. The initial 

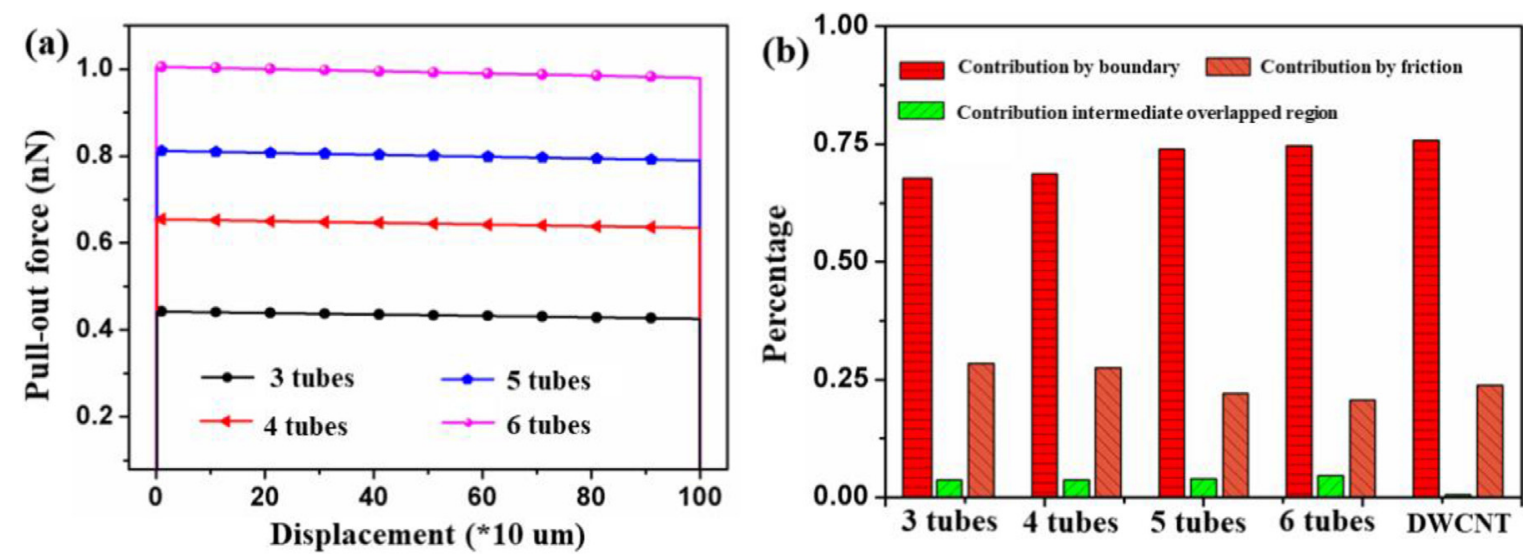

Fig. 13. The pull-out process of CNTB and the components of the pull-out force. (a) shows pull-out force variation when the core tube is pulled out from CNTBs with different CNT number. (b) expresses the proportion of components of the pull-out force indicating the maximum contribution comes from the boundary.

length of the CNTB is $2 \mathrm{~mm}$ and the pull-out velocity is 5.6-7.2 $\mu \mathrm{m} / \mathrm{s}$. To compare with the experiment data and the obtained results of ultra-long DWCNT, the computation system is also chosen as $((9,0) @(10,10) s)$, where the length is $1 \mathrm{~mm}$ and the pull-out velocity is $7 \mu \mathrm{m} / \mathrm{s}$. The pull-out process of CNTB is calculated in Fig. 13 (a) and components of the pull-out force are shown in Fig. 13 (b). The pull-out behaviors of the CNTB consisting of three tubes are close to that of the DWCNT on nanoscale in Fig. 9(b), where the pull-out force slightly decreases in the second stage. However, an obvious decline of pull-out force can be observed for the CNTB composed of six tubes with the core tube being pulled out, which is same as that of the ultra-long DWCNT in Fig. 11(a). This attributes to the increasing dynamic friction coefficient for the CNTB with more tubes, which is illustrated in Fig. 6(d). In addition, compared with the results of the DWCNT when the pull-out velocity is $7 \mu \mathrm{m} / \mathrm{s}(1.1 \mathrm{nN})$, the pull-out force of the CNTB is obviously smaller $(0.5-1.0 \mathrm{nN})$. The reason for this phenomenon can be traced to Fig. 6(c) that the adhesion energy increases with the tube number in the CNTB increasing, while it is the maximum for the DWCNT because of the largest contact area.

Moreover, as shown in Fig. 13 (b), the pull-out force is still mainly contributed by the boundary effects and the percentage is seventy, although the contribution of the dynamic friction force reaches the same order of magnitude accounting for 25 percent. Therefore, with increasing the CNT number, the boundary effect shows upward tendency, while the contribution of the dynamic friction force decreases. Another important result can be obtained from Fig. 13 (b) that the contribution from the intermediate overlapped region is greatly improved and it maintains nearly 5 percent for ultra-long CNTB, while the percentage is 0.5 percent for the ultra-long DWCNTB. This attributes to the commensurate configuration of the CNTB in the circumferential direction, as illustrated in Fig. 6(e) and 7(c). Thus, the contribution of this part cannot be ignored anymore.

Based on the results obtained above, the mechanism behind the experiment results measured in Section 2 of the interaction in the CNTB can be properly predicted. Compared to the pull-out force of ultra-long DWCNT (7.8 nN when the pull-out velocity is 6.9-10.2 $\mu \mathrm{m} / \mathrm{s})$, it is obviously smaller for CNTB $(0.8 \mathrm{nN}$ when the pull-out velocity is $5.6-7.2 \mu \mathrm{m} / \mathrm{s})$. This attributes to the decreased adhesion energy for the CNTB with smaller contact area. Thus, the boundary effect is weakened, which is the main contribution to the pull-out force. This mechanism is also verified by the computation based on the multi-scale model. Moreover, the pull-out force tested is higher than the previous reported values in the quasi-static state, which attributes to the fact that the dynamic friction force cannot be ignored in our experiment.

\section{Conclusions}

This paper addresses the interaction in millimeters long CNT/CNTB by conducting experiment and numerical analysis. The innovative experiment device is setup to test the pull-out behaviors of these special structures, which are macroscale in axial direction and nanoscale in the circumferential direction, respectively. By attaching the force senor to the probe, the continuous displacement-loading relationship can be recorded timely and the inter-wall/inter-tube interaction is firstly insitu tested in OM. Moreover, the bottom-up approach is adopted to carry on the multi-scale analysis. The basic parameters of the CNT/CNTB are obtained by the MD simulation, based on which the coarse-grained model can be obtained. According to the framework of Frenkel-Kotorova, the macroscale model can be established, where the boundary effects, the dynamic friction and the commensurability are considered. Therefore, results obtained in our model can be directly compared with the experimental data. Also, the mechanism for the experiment phenomena can be explained well. In addition, based on our model, we can make some predictions of the interaction in ultra-long CNT/CNTB. Several important conclusions are drawn as follows. 
(1) The pull-out force consists of three parts, including the boundary effect, the dynamic friction and the configuration force. The boundary effect is the main component and is related to the contact area at boundary. The dynamic friction force originates from phonon dissipation through the interaction between inner/core tube and outer/side one when the system temperature reaches $300 \mathrm{~K}$. Contribution from configuration force is related to the commensurability of the structure, which cannot be ignored anymore when the commensurate configuration appears.

(2) For the DWCNT on nanoscale, the contribution of the pull-out force from the dynamic friction and the intermediate overlapped area is much smaller than that of the boundary effect, resulting in the independent of the overlapped region. However, when the pull-out velocity is $12 \mu \mathrm{m} / \mathrm{s}$ for a one-millimeter-long DWCNT/CNTB, the boundary effect is still the main component, which can account for nearly $70 \%$, while the contribution of the dynamic friction force reaches the same order of magnitude accounting for $25 \%$.

(3) The linear dependence of the pull-out force on the velocity applied on the inner/core tube is determined by the dynamic friction force, which is the product of the velocity and the dynamic friction coefficient. The dynamic friction coefficient is proved to be 0.29 multiple of critical.

(4) According to the experiment results, the pull-out force of the CNTB is far less than that of the DWCNT. This mainly attributes to the smaller boundary interaction force for the CNTB, where the contact area greatly decreases.

(5) The pull-out force tested in the experiment is higher than the previous reported values because the dynamic friction in the millimeter-long DWCNT/CNTB cannot be ignored anymore when the pull-out velocity reaches a few to a dozen microns per second.

\section{Declaration of Competing Interests}

The authors declare that they have no known competing financial interests or personal relationships that could have appeared to influence the work reported in this paper.

\section{Acknowledgments}

We gratefully acknowledge support from the National Natural Science Foundation of China (Grant nos. 11872035, 11902311, 11632010, 51872156 and 21636005), Foundation for the National Basic Research Program of China (Grant No. 2016YFA0200102), the Beijing Municipal Science and Technology Commission (Grant No. D141100000614001).

\section{Supplementary materials}

Supplementary material associated with this article can be found, in the online version, at doi:10.1016/j.jmps.2020. 104032.

\section{References}

Akita, S., Nakayama, Y., 2003a. Interlayer sliding force of individual multiwall carbon nanotubes. Jpn. J. Appl. Phys. 42, $4830-4833$.

Akita, S., Nakayama, Y., 2003b. Extraction of inner shell from multiwall carbon nanotubes for scanning probe microscope tip. Jpn. J. Appl. Phys. 42, 3933-3936.

Akita, S., Nakayama, Y., 2005. Mechanical and electrical properties of multiwall nanotube under interlayer sliding. e-J. Surf. Sci. Nanotechnol. 3, 86-93.

Bai, Y., Zhang, R., Ye, X., Zhu, Z., Xie, H., Shen, B., Zhang, S., 2018. Carbon nanotube bundles with tensile strength over 80 GPa. Nat Nanotechnol. 13 , 589.

Benassi, A., Ma, M., Urbakh, M., Vanossi, A., 2015. The breakdown of superlubricity by driving-induced commensurate dislocations. Sci. Rep. 5, 16134.

Braun, O.M., Kivshar, Y.S., 2004. The Frenkel-Kontorova models springer. Berlin. Phys. Rep. $3061998 \mathrm{~d}$.

Buehler, Markus, J., 2006. Mesoscale modeling of mechanics of carbon nanotubes: self-assembly, self-folding, and fracture. J Mat Res. 21, $2855-2869$.

Cumings, J., Zettl, A., 2000. Low-friction nanoscale linear bearing realized from multiwall carbon nanotubes. Science 289, 602-604.

Clough, R.W., Penzien, J.M., 2003. Dynamics of Structures. New York: Computers \& Structures. Inc., USA.

Cook, E.H., Buehler, M.J., Spakovszky, Z.S., 2013. Mechanism of friction in rotating carbon nanotube bearings. J Mech Phys Solids 61, $652-673$.

Fennimore, A.M., Yuzvinsky, T.D., Han, W.Q., Fuhrer, M.S., Cumings, J., Zettl, A., 2003. Rotational actuators based on carbon nanotubes. Nature 424, 408-410.

Filleter, T., Yockel, S., Naraghi, M., Paci, J.T., Compton, O.C., Mayes, M.L., Espinosa, H.D., 2012. Experimental-computational study of shear interactions within double-walled carbon nanotube bundles. Nano Lett. 12, 732-742.

Gabor, D., 1946. Theory of communications. J. Inst. Electr. Eng. 93, 429-457.

Guo, Y., Guo, W., 2003. Mechanical and electrostatic properties of carbon nanotubes under tensile loading and electric field. J. Phys. D 36, 805-811.

Guo, W., Guo, Y., Gao, H., Zheng, Q., Zhong, W., 2003. Energy dissipation in gigahertz oscillators from multiwalled carbon nanotubes. Phys. Rev. Lett. 91 125501-1-125501-4.

Guo, W., Gao, H., 2005. Optimized bearing and interlayer friction in multiwalled carbon nanotubes. Comput. Model. Eng. Sci. 7, 19-34.

Guo, W., Zhong, W., Dai, Y., Li, S., 2005. Coupled defect-size effects on interlayer friction in multiwalled carbon nanotubes. Phys. Rev. B (Conden. Matter Mater. Phys.). 72, 075409-075410.

Halgren, T.A., 1992. The representation of van der Waals (vdW) interactions in molecular mechanics force fields: potential form, combination rules, and vdW parameters. J. Am. Chem. Soc. 114, 7827-7843.

Hepplestone, S.P., Srivastava, G.P., 2006. Phonon-phonon interactions in single-wall carbon nanotubes. Phys. Rev. B 74, 165420.

Kis, A., Jensen, K., Aloni, S., Mickelson, W., Zettl, A., 2006. Interlayer forces and ultralow sliding friction in Energy Dissipation in carbon nanotubes. Phys. Rev. Lett. 97, 025501

Krylov, S.Y., Dijksman, J.A., Van Loo, W.A., Frenken, J.W.M., 2006. Stick-slip motion in spite of a slippery contact: do we get what we see in atomic friction? Phys. Rev. Lett. 97, 166103.

Krylov, S.Y., Frenken, J.W.M., 2012. The problem of critical damping in nanofriction. Colloid J. 74, 569-572.

Li, Y., Hu, N., Yamamoto, G., Wang, Z., Hashida, T., Asanuma, H., Fukunaga, H., 2010. Molecular mechanics simulation of the sliding behavior between nested walls in a multi-walled carbon nanotube. Carbon. 48, 2934-2940.

Liu, L., Ma, W., Zhang, Z., 2011. Macroscopic carbon nanotube assemblies: Preparation, properties, and potential applications. Small 7, $1504-1520$. 
Liu, P., Zhang, Y.W., 2011. A theoretical analysis of frictional and defect characteristics of graphene probed by a capped single-walled carbon nanotube. Carbon. 49, 3687-3697.

Liu, Y., Hong, J., Zhang, Y., Cui, R., Wang, J., Tan, W., Li, Y., 2009. Flexible orientation control of ultralong single-walled carbon nanotubes by gas flow. Nanotechnology 20, 185601.

Lu, W., Zu, M., Byun, J.H., Kim, B.S., Chou, T.W., 2012. State of the art of carbon nanotube fibers: Opportunities and challenges. Adv. Mat. $24,1805-1833$.

Mirzaeifar, R., Qin, Z., Buehler, M.J., 2015. Mesoscale mechanics of twisting carbon nanotube yarns. Nanoscale 7, 5435-5445.

Müser, M.H., 2002. Nature of mechanical instabilities and their effect on kinetic friction. Phys. Rev. Lett. 89, 224301.

Nosé, S., 1984. A unified formulation of the constant temperature molecular dynamics methods. J. Chem. Phys. 81, 511-519.

Omata, Y., Yamagami, Y., Tadano, K., Miyake, T., Saito, S., 2005. Nanotube nanoscience: a molecular-dynamics study. Phys. E 29, 454-468.

Paci, J.T., Furmanchuk, A.O., Espinosa, H.D., Schatz, G.C., 2016. Shear and friction between carbon nanotubes in bundles and yarns. Nano Lett. 14, 6138.

Plimpton, S., 1995. Fast parallel algorithms for short-range molecular dynamics. J. Comp. Phys. 117, 1-19.

Prasad, M.V.D., Bhattacharya, B., 2017. Phononic origins of friction in carbon nanotube oscillators. Nano Lett. 17, $2131-2137$.

Riedo, E., Chevrier, J., Comin, F., Brune, H., 2001. Nanotribology of carbon based thin films: the influence of film structure and surface morphology. Surf. Sci. 477, 25-34.

Rivera, J.L., McCabe, C., Cummings, P.T., 2003. Oscillatory behavior of double walled nanotubes under extension: a simple nanoscale damped spring. Nano Lett. 3, 1001-1005.

Rivera, J.L., McCabe, C., Cummings, P.T., 2005. The oscillatory damped behaviour of incommensurate double-walled carbon nanotubes. Nanotechnology 16, $186-198$.

Savitzky, A., Golay, M.J., 1964. Smoothing and differentiation of data by simplified least squares procedures. Anal. Chem. 36, $1627-1639$.

Servantie, J., Gaspard, P., 2006a. Rotational dynamics and friction in double-walled carbon nanotubes. Phys. Rev. Lett. 97 186106-1-186106-4.

Servantie, J., Gaspard, P., 2006b. Translational dynamics and friction in double-walled carbon nanotubes. Phys. Rev. B 73, 125428.

Stuart, S.J., Tutein, A.B., Harrison, J.A., 2000. A reactive potential for hydrocarbons with intermolecular interactions. J. Chem. Phys. 112, 6472-6486.

Suekane, O., Nagataki, A., Mori, H., Nakayama, Y., 2008. Static friction force of carbon nanotube surfaces. Appl. Phys. Express 1 , 064001.

Urbakh, M., Meyer, E., 2010. Nanotribology: the renaissance of friction. Nat. Mater. 9, 8.

Yakobson, B.I., Samsonidze, G., Samsonidze, G.G., 2000. Atomistic theory of mechanical relaxation in fullerene nanotubes. Carbon. 38, 1675-1680.

Ye, X., Cui, Z., Fang, H., Li, X., 2017a. A multiscale material testing system for in situ optical and electron microscopes and its application. Sensors 17, 8.

Ye, X., Zhao, L., Liang, J., Li, X., Chen, G.Q., 2017b. Study of the tensile properties of individual multicellular fibres generated by Bacillus subtilis. Sci. Rep. 7, 46052.

Yu, M., Lourie, O., Dyer, M.J., Moloni, K., Kelly, T.F., Ruoff, R.S., 2000a. Strength and breaking mechanism of multiwalled carbon nanotubes under tensile load. Science 287, 637-640.

Yu, M., Yakobson, B.I., Ruoff, R.S., 2000b. Controlled sliding and pullout of nested shells in individual multiwalled carbon nanotubes. J. Phys. Chem. B. 104, 8764-8767.

Yuan, X., Wang, Y., 2017. Atomistic simulations on interwall sliding behaviour of double-walled carbon nanotube: effects of structural defects. Mol. Simul. 43, 953-961.

Yuan, X., Wang, Y., 2018. Adhesion of carbon nanotubes on elastic substrates with finite thickness. J. Appl. Phys. 124, 155306.

Zhang, S., Liu, W.K., Ruoff, R.S., 2004. Atomistic simulations of double-walled carbon nanotubes (DWCNTs) as rotational bearings. Nano Lett. 4, $293-297$.

Zhang, R., Zhang, Y., Zhang, Q., Xie, H., Wang, H., Nie, J., Wei, F., 2013c. Optical visualization of individual ultralong carbon nanotubes by chemical vapour deposition of titanium dioxide nanoparticles. Nat. Commun. 4, 1727.

Zhang, S., Zhao, S., Xia, M., Zhang, E., Xu, T., 2003. Ring formation of single-walled carbon nanotubes: competition between conformation energy and entropy. Phys. Rev. B 68, 5419.

Zhang, R., Ning, Z., Xu, Z., Zhang, Y., Xie, H., Ding, F., Chen, Q., Zhang, Q., Qian, W., Cui, Y., Wei, F., 2016. Interwall friction and sliding behavior of centimeters long double-walled carbon nanotubes. Nano Lett. 16, 1367-1374.

Zhang, R., Ning, Z., Zhang, Y., Zheng, Q., Chen, Q., Xie, H., 2013a. Superlubricity in centimetres-long double-walled carbon nanotubes under ambient conditions. Nat. Nanotech. 8, 912-916.

Zhang, R., Zhang, Y., Wei, F., 2017a. Controlled synthesis of ultralong carbon nanotubes with perfect structures and extraordinary properties. Acc. Chem. Res. 50, 179-189.

Zhang, R., Zhang, Y., Wei, F., 2017b. Horizontally aligned carbon nanotube arrays: growth mechanism, controlled synthesis, characterization, properties and applications. Chem. Soc. Rev. 46, 3661-3715.

Zhang, R., Zhang, Y., Zhang, Q., Xie, H., Qian, W., Wei, F., 2013b. Growth of half-meter long carbon nanotubes based on Schulz-Flory distribution. ACS Nano 7, 6156-6161.

Zhao, Y., Ma, C.C., Chen, G., Jiang, Q., 2003. Energy dissipation mechanisms in carbon nanotube oscillators. Phys Rev Lett. $91,175504$.

Zheng, Q., Jiang, Q., 2002. Multiwalled carbon nanotubes as gigahertz oscillators. Phys. Rev. Lett. 88, 045503.

Zheng, Q., Liu, J.Z., Jiang, Q., 2002. Excess van der Waals interaction energy of a multiwalled carbon nanotube with an extruded core and the induced core oscillation. Phys. Rev. B 65, 245409. 